\title{
Un cercueil dans la cour : des funérailles évangéliques dans une famille transnationale haïtienne
}

A coffin in the courtyard: evangelical funerals in a transnational Haitian family Um caixão no quintal: funerais evangélicos em uma família haitiana transnacional

\section{Nadège Mézié}

\section{(2) OpenEdition Journals}

Édition électronique

URL : https://journals.openedition.org/jsa/19310

DOI : 10.4000/jsa. 19310

ISSN : 1957-7842

\section{Éditeur}

Société des américanistes

Édition imprimée

Date de publication : 30 septembre 2021

Pagination : 9-43

ISSN : 0037-9174

Référence électronique

Nadège Mézié, "Un cercueil dans la cour : des funérailles évangéliques dans une famille transnationale haïtienne ", Journal de la Société des américanistes [En ligne], 107-1 | 2021, mis en ligne le 30 septembre 2021, consulté le 02 septembre 2022. URL : http://journals.openedition.org/jsa/ 19310 ; DOI : https://doi.org/10.4000/jsa.19310 


\title{
Un cercueil dans la cour : des funérailles évangéliques dans une famille transnationale haïtienne
}

\author{
Nadège MÉzIÉ *
}

\begin{abstract}
Si les funérailles en milieu vodou haïtien ont fait l'objet de l'attention des anthropologues, on sait peu de choses sur la manière dont elles sont organisées et réalisées par les familles et les églises évangéliques. Cet article propose une étude détaillée d'un cas ethnographique de funérailles évangéliques, objet d'un coup de théâtre, dans les mornes du sud-ouest d'Haïti au sein d'une famille dont de nombreux membres appartiennent à la diaspora. La première partie décrit le rituel, depuis le traitement du corps du défunt jusqu'au culte d'enterrement. La deuxième partie analyse ces funérailles et en particulier les conflits auxquelles elles donnent lieu, à travers deux dynamiques sociales qui s'y donnent à voir, celle du changement religieux avec la conversion massive de populations rurales au protestantisme évangélique et pentecôtiste et celle de la migration avec, dans la plupart des familles haïtiennes, des parents vivant à l'étranger. [Mots-clés: Haïti, funérailles, églises évangéliques, parenté, diaspora, conflits.]
\end{abstract}

A coffin in the courtyard: evangelical funerals in a transnational Haitian family. While Haitian vodou funerals have been studied extensively by anthropologists, little is known about how they are organized and carried out by evangelical families and churches. This article offers a detailed case study of an evangelical funeral in the mountains of southwestern Haiti, in a family with many members of the diaspora. The first part details the ritual, from the treatment of the corpse to the burial service. The second part offers an analysis of the funerals, and in particular the conflicts that have arised as a result of two broader social dynamics: the religious change in the country, with the massive conversion of rural populations to Evangelical and Pentecostal Protestantism, and the migrations, which led to most Haitian families having at least one member living abroad. [Keywords: Haiti, funerals, evangelical churches, kinship, diaspora, conflicts.]

Um caixão no quintal: funerais evangélicos em uma família haitiana transnacional. Se os funerais entre praticantes do vodu no Haiti foram bem documentados por antropólogos, pouco sabemos sobre sua organização e desenrolar em meio

* FE/Unicamp, Campinas (Brasil) [nagmezie@gmail.com]. 
evangélico. Apresento aqui o caso etnográfico de um funeral realizado nas montanhas do sudoeste do Haiti, em uma família cujos membros são em sua maioria evangélicos e muitos pertencem à diáspora. Na primeira parte do artigo descrevo o ritual, desde as técnicas de tratamento do corpo da pessoa falecida até o culto durante o enterro. Na segunda parte, analiso o funeral, e em particular os conflitos que dele decorrem, à luz de duas dinâmicas sociais mais amplas: a das transformações na paisagem religiosa do país, com a conversão massiva das populações rurais ao protestantismo evangélico e pentecostal, e a da intensificação da migração ao estrangeiro, materializada no fato de que, atualmente, todas as famílias haitianas ou quase têm pelo menos um de seus membros morando fora do país. [Palavras chaves: Haiti, funerais, igrejas evangélicas, parentesco, diáspora, conflitos.]

La mort, les relations entre les morts et les vivants et les rituels funéraires en Haïti ont fait l'objet d'un certain nombre de travaux qui, pour leur grande majorité, les ont explorés tels qu'ils se manifestent dans l'univers vodou ${ }^{1}$. On aura, cependant, quelque peine à trouver dans la littérature anthropologique consacrée à Haïti des descriptions ethnographiques, détaillées, situées et récemment produites, de funérailles vodou qu'elles soient rurales ou urbaines et plus encore de funérailles exclusivement catholiques ou évangéliques. Les annotations de Métraux (1954 et 1958, p. 216-228), mais également celles de Suzanne Comhaire-Sylvain $(1959)^{2}$, sur les cérémonies funéraires en milieu vodou, d'une grande richesse mais peu indexées, sont aujourd'hui à prendre pour ce qu'elles sont, à savoir un matériau historiquement daté qui doit être révisé à l'aune des pratiques funéraires contemporaines, à la manière dont Karen Richman a pu le faire en traitant des «nouveaux rites mortuaires de passage » (2018 [2005], p. 124-128; 2018) ou encore Paul Farmer en observant la pluralité des traitements thérapeutiques, les interprétations de la maladie et les funérailles de personnes atteintes du sida (1992).

Le constat de Joël Noret, appliqué à l'Afrique subsaharienne, selon lequel les études consacrées à la place des morts « sont restées attachées trop exclusivement

1. On trouve un état de l'art sur la question chez Lowenthal 1987, p. 228-245. J'ajoute, ici, des références plus récentes: pour les cérémonies funéraires et, plus largement, pour le culte des morts: Richman (2018 [2005] et 2018); pour Baron Samedi et la cohorte des autres Gedes, lwa (esprit) dont le domaine d'action est la mort et la sexualité: Desmangles (1992, p. 114-124), Hurbon (1993, p. 94-95), McCarthy Brown (2001 [1991], p. 330-368), Smith (2012); et pour leur actuation dans le cimetière principal de Port-au-Prince: McCarthy Brown (2001 [1991], p. 368-372), Smith (2010); pour la figure du zombie: McAlister (2002, p. 102-111), Charlier (2015); et pour les représentations abondantes de la mort, de Baron Samedi et des Gedes, dans l'art et les objets de culte: Cosentino (2012 et 2018) et Braziel (2017).

2. Suzanne Comhaire-Sylvain a consacré de nombreuses notes de terrain, jamais publiées, aux rites funéraires (https://oac.cdlib.org/findaid/ark:/13030/c8k074wr/entire_text/, consulté le $01 / 07 / 2021)$. 
aux cultures [...] "traditionnelles" » (2004, p. 143), peut être transposé à ce qui a été produit sur la question en Haïti, où le vodou a longtemps été l'objet privilégié, pour ne pas dire fétiche, des chercheurs. Ce tropisme a contribué à retarder l'intérêt des anthropologues et des sociologues pour l'implantation des protestantismes évangéliques et pentecôtistes, auxquels Métraux, malgré l'inclination teintée de fascination pour le vodou qu'il partageait avec ses contemporains, avait pourtant pu être sensible dès les années $1940^{3}$. Les funérailles évangéliques ont, par conséquent, échappé à l'attention des chercheurs.

Cet article prétend, en premier lieu, combler ce vide en décrivant avec soin un cas ethnographique de funérailles évangéliques en milieu rural haïtien, au sein d'une famille dont les enfants ont tous migré à l'étranger. En Haïti, les transformations religieuses sont intrinsèquement liées à la dynamique migratoire, lesquelles contribuent à reconfigurer les interdépendances familiales et les relations entre milieu rural et milieu urbain. Le rituel des funérailles se trouve ainsi soumis à des transformations qui affectent le sens qui lui est attribué et les manipulations qui y ont cours. Il doit aussi répondre à de nouvelles exigences en raison de l'éclatement résidentiel des membres de la parenté. Prêter attention aux funérailles contemporaines haïtiennes, c'est pouvoir accéder aux arrangements familiaux, économiques et religieux qui s'opèrent, de manière microsociologique, à la faveur de mouvements mineurs et majeurs d'ajustements, de concessions, de négociations et de conflits et qui témoignent de dynamiques sociales de grande échelle. Pour Claudine Vidal (1991, p. 87-98) et Joël Noret (2017; Jindra et Noret 2011), dans la lignée de l'anthropologie dynamique de Georges Balandier, les funérailles doivent être traitées comme des « révélateurs » de transformations sociales, d'enjeux et de « relations de pouvoir et de dépendance » (Vidal 1991, p. 87). Dans une perspective inspirée de ces travaux, j'explorerai donc en second lieu l'entrelacement des dynamiques religieuses et migratoires, telles qu'elles se manifestent de manière singulière et située au cours des funérailles décrites dans la première partie. Nous verrons comment elles façonnent l'organisation du rituel - aussi bien dans sa temporalité que dans les actes de son exécution (gestes et paroles) - et la distribution conflictuelle des rôles des participants. Il apparaît que le rituel des funérailles peut aussi bien conforter la cohésion du groupe endeuillé qu'introduire ou renforcer les dissensions et les conflits. Comme le souligne Yvon Van der Pijl, il est nécessaire d' " aborder les rites de passage comme des pratiques dynamiques, parfois

3. Métraux écrit en 1953: «Le succès des sectes protestantes est un phénomène sociologique et psychologique d'un grand intérêt qui mériterait une attention spéciale » (1953, p. 199). Voir également les mentions du protestantisme dans Le vaudou haïtien (1958, voir en particulier p. 311-317). Melville Herskovits fait également mention au succès grandissant des « sectes protestantes » (2012 [1937], p. 290-291) dans son livre Life in a Haitian Valley reposant sur un terrain effectué dans la vallée de Mirebalais en 1934. 
désintégratrices, plutôt que comme de simples manifestations et des fortifiants des liens sociaux et valeurs culturelles » (2016, p. 149). Si les funérailles reposent sur la solidarité familiale et la donnent à voir, elles sont aussi le théâtre de divisions et de conflits - en raison précisément des enjeux d'exhibition, de libéralité, de statut social et d'héritage, ainsi que des luttes qui portent sur la définition de qui est et qui n'est pas parent (De Witte 2001, p. 51-80; Van der Pijl 2016; Vidal 1991, p. 87-98; Smith 2004).

\section{Les funérailles de Grann Vierge}

\section{La mort de Grann}

Grann Geneviève, plus souvent appelée Grann Vierge ou tout simplement Grann ${ }^{4}$, a vécu toute sa vie dans les mornes du département de la Grand'Anse, situé dans le sud-ouest d'Haïti. Sa famille appartenait à la paysannerie, elle était relativement bien dotée en terres, possédait plusieurs têtes de bétail, et avait coutume de résider dans un bourg ou dans ses environs. Grann devint à 21 ans la deuxième femme d'un spéculateur de café, propriétaire de plusieurs carreaux de terre ${ }^{5}$, et petit notable qui fut maire de la ville côtière de Pestel. Il lui fit construire, dans le lieu-dit de Mòn Anvè, une maison de trois pièces en bord de route sur des terres qu'il possédait, à une dizaine de kilomètres de sa résidence, et lui rendait visite régulièrement ${ }^{6}$. Grann, qui possédait un petit capital social par ses consanguins, gagna, avec ce plasaj (union coutumière qui autorise la polygamie), en reconnaissance sociale et assura à chacun des descendants de

4. Prénoms et localités ont été rendus anonymes. Quand une femme est âgée, elle est souvent appelée Grann (terme qui signifie grand-mère) suivi de son prénom ou de son surnom.

5. Un carreau de terre équivaut à un peu plus de 1,29 hectares. Le conjoint de Grann avait donc été épargné par la crise du système paysan caractérisé par le morcellement des terres (Ans 1987).

6. Il n'était pas rare, pour un homme possédant suffisamment de terres de maintenir au moins deux foyers, parfois éloignés de plusieurs kilomètres l'un de l'autre, avec chacun à leur tête une femme. Dans les mornes, le deuxième foyer pouvait se trouver sur une parcelle de terres dans les hauteurs et l'homme circulait entre ces deux foyers. La femme à qui était assigné le maintien sur les terres de culture était souvent appelée « femme-jardin ». Les ressources allouées par l'homme à la femme installée au bourg, en bord de route ou sur des terres moins isolées étaient plus élevées que celles attribuées à la « femme-jardin ». Ce qualificatif n'avait pas été attribué à Grann Vierge en raison du statut social de ses parents consanguins. Plusieurs personnes qui l'avaient connue m'ont affirmé que Grann l'aurait de toute façon refusé et que, par ailleurs, elle avait su s'imposer comme l'égal de sa matelot du bourg (en créole, «matelote » désigne une coépouse). La pluri-conjugalité instituée, en raison de l'appauvrissement de la paysannerie, de la « parcellisation des terres » (Ans 1987, p. 221-234), mais aussi des injonctions moralisantes des églises évangéliques et pentecôtistes, est de plus en plus rare aujourd'hui. 
cette relation un lot de terres qui les prémunit de la misère ${ }^{7}$. À l'âge de 62 ans, elle devint veuve, ses enfants étaient tous partis depuis plusieurs années déjà à l'étranger, elle continuait à cultiver ses terres. À cette époque, un de ses frères aîné était venu la rejoindre. En 2006, elle avait alors 80 ans, sa santé se mit à se détériorer. Au cours de cette même année, presque tous les jours, je la retrouvais dans sa cuisine où, bien qu'affaiblie, elle n'en continuait pas moins de railler ses voisins, de bougonner contre l'absence de ses enfants, pour l'instant d'après se féliciter de les savoir loin de ce bout d'île « où les hommes se haïssent (nèg rayi nèg) $\|^{8}$. Plusieurs fois, je l'ai entendue adresser des récriminations sévères à l'encontre de ses voisins immédiats, un couple de missionnaires évangéliques provenant des États-Unis à qui elle avait vendu une grande partie de ses terres 15 ans plus tôt. Ils y avaient installé une antenne de l'Evangelical Bible Mission (EBM), mission non-dénominationnelle aux forts accents baptistes, dont la maison-mère se trouve en Floride. Le petit-fils de Grann, Olsen, aime à rappeler que personne d'autre que Grann n'avait accepté de leur vendre des terres. Cette vente introduisit Olsen, alors âgé de 17 ans, auprès des missionnaires, qui l'employèrent, pour des tâches plus ou moins longues, tout le temps de l'activité de la mission, jusqu'à sa fermeture en 2010, et firent de lui un pilier de la mission et un leader évangélique respecté. Dès les premières semaines de leur installation, les missionnaires furent rejoints par une mère et ses trois filles adolescentes. L'une d'elle devint la femme d'Olsen. Les deux autres épousèrent des hommes qui occupèrent ensuite des positions de leaders au sein de la mission. Tous intégrèrent le comité de l'église, sur lequel ils régnèrent pendant quinze ans, et furent employés, à un moment ou un autre, par les missionnaires. Les premiers mois de l'implantation de la mission baptiste, Grann assista au culte dominical. Elle refusa néanmoins de se convertir pour ne pas abandonner les saints catholiques auxquels elle avait voué un culte sa vie durant ${ }^{9}$ et pour continuer à se défendre activement (à travers des pratiques empruntées au vodou) contre le malheur et les persécutions. Son entêtement n'avait pas été du goût des missionnaires et Olsen croit savoir qu'un jour une dispute éclata au cours de laquelle Grann leur dit qu'elle regrettait de leur avoir vendu ses terres à un prix très en-dessous de ceux qui avaient cours. Elle s'enorgueillissait de dire, quand je l'ai connue, qu'elle n'avait plus jamais passé le portail de la mission

7. Formellement, le partage foncier est égalitaire entre hommes et femmes et entre enfants naturels et enfants légitimes. Dans les faits, des héritiers sont tenus à l'écart ou s'auto-excluent (par l'exode en ville ou la migration à l'étranger).

8. Entre juillet 2005 et février 2007, j'ai enquêté, dans le cadre de mon doctorat en anthropologie, dans les mornes du département de la Grand'Anse et ai été hébergée, durant tout ce temps, par Olsen, le petit-fils de Grann Vierge, et sa femme.

9. En Haïti, la «symbiose par identification » (Desmangles 1992, p. 10) a conduit à ce que, sur la base de similarités, les saints catholiques soient identifiés ou «transfigurés » dans des esprits vodou. 
EBM et, dans le même temps, elle reprochait aux missionnaires de l'avoir délaissée une fois les terres acquises et de ne s'être jamais déplacés jusqu'à elle. «Ils ne m'ont pas montré de respect (Yo pa fè respè) », m’avait-elle dit au cours de l'une de nos conversations et, dans une autre, « ils croient que je suis riche parce que j'ai un peu de terre (paske m'gen kèk tè, yo kwe se rich, $m$ 'rich) ». Olsen et sa femme avaient tenté vainement d'obtenir sa conversion et mettaient ses impiétés sur le compte de l'âge («C'est des choses de vieilles personnes (Se bagay granmoun) »). Les derniers mois de sa vie, elle se disait persécutée par l'esprit de son conjoint décédé. Pour le faire fuir, elle s'habillait chaque jour de rouge, ses draps étaient de la même couleur et elle avait placé, sous son lit, un giraumon (courge), réputé éloigner les mauvais esprits. Quand elle fût très affaiblie, elle appela un hougan (spécialiste vodou du rituel et de la guérison). Cependant, elle ne refusait pas les prières et l'imposition des mains de parents, d'amis et de fidèles évangéliques ou pentecôtistes qui lui rendaient visite (Mézié 2019a). Ce pluralisme médico-religieux, répandu en Haïti (Vonarx 2008), avait été complété par des allers-retours à 1'hôpital des Cayes, sous l'égide d'Olsen, le seul de ses petits-enfants à être resté dans les mornes, où les médecins lui avaient diagnostiqué une insuffisance cardiaque. Madan Olsen, l'épouse d'Olsen ${ }^{10}$, secondait son mari dans les soins prodigués à Grann: elle lui envoyait chaque jour ou presque à manger.

En décembre 2006, l'état de Grann s'aggrava. Début janvier 2007, un aprèsmidi, elle succomba. Le lendemain matin, la plupart des meubles et des objets avaient déjà été enlevés et stockés dans une pièce fermée à clef. Une vingtaine de femmes évangéliques, pentecôtistes ${ }^{11}$, mais aussi serviteurs de $l w a^{12}$ et/ou catholiques, dont plusieurs étaient des habituées du lavage des défunts, étaient à son chevet. Madan Olsen était au milieu d'elles. Comme en beaucoup d'autres endroits au monde, c'est aux femmes que revient la manipulation des corps (Bloch et Parry 1982, p. 22-27). Métraux avait déjà constaté que c'était aux « vieilles femmes » qu'était attribuée la tâche de la toilette mortuaire, et qu'elles pouvaient « sans manquer à la pudeur, s'occuper du cadavre masculin » (1954, p. 290).

10. L'épouse ou concubine est appelée par le prénom du mari précédé par Madame (Madan en créole haïtien).

11. Les fidèles affiliés à cette branche du protestantisme ont coutume de se dire kretyen (chrétien) mais ils peuvent aussi parfois se définir en fonction de la dénomination ou du courant auquel se rattache leur église (on peut ainsi se dire pantekotis, evangelik, advantis - pentecôtiste, évangélique, adventiste). Sur les autodénominations religieuses en fonction des situations voir Mézié (2019b). Je parle ici de femmes évangéliques et pentecôtistes qui se réfèrent à leur affiliation à des églises dont la forme du culte et la doctrine professée diffèrent de manière perceptible par les fidèles. Les pentecôtistes diront que, chez eux, « c'est plus chaud» (ils font référence aux musiques et aux danses plus rythmées et moins contenues que dans le culte évangélique) et chez les évangéliques «c'est plus froid ».

12. Esprits du vodou. Les pratiquant du vodou sont nommés « serviteurs de lwa». 
Le dernier souffle de Grann laissa place à la confusion. Pendant une trentaine de minutes, on se disputa pour savoir si elle était effectivement morte ou simplement évanouie: « Son ventre continue de bouger (Vant la ap kontinye monte desann) », " Non, c'est le lit qui bouge (Non, se kabann nan k'ap bouje) », « S'il vous plaît, sortez de la chambre! (Tanpri, kite chanm nan, rale kò nou!) ». Une voisine se vit rabrouée alors qu'elle se préparait à pousser un premier rèl (pleurs, cris et lamentations mêlés): «Fais pas ça! On n’a pas fermé sa bouche (Pa fè sa non! Bouch li pa mare) ». L'infraction aurait valu aux parents de Grann de la suivre bientôt dans la tombe. La toilette mortuaire n'a pas été, ou presque, modifiée dans les milieux évangéliques et pentecôtistes des mornes du département de la Grand'Anse: à peu de choses près, elle se déroule de la même manière que lorsqu'elle est réalisée pour un défunt qui aurait été sa vie durant pratiquant du vodou, donnant ainsi une coloration syncrétique aux funérailles évangéliques. Les femmes, qui ont pour habitude d'accompagner et de réaliser la toilette, sont dépositaires d'un savoir et d'une praxis qui relève du rite catholico-vodou et elles interviennent dans tous les milieux religieux, à la manière des matrones (sages-femmes qui exercent en-dehors des structures biomédicales) qui sont appelées dans les familles évangéliques ou pentecôtistes et déploient un ensemble de pratiques prophylactiques et thérapeutiques qui sont partagés dans le vodou (Tremblay 1995; Damus 2020). Les femmes qui sont mobilisées pour opérer des soins au cours des différents cycles de la vie sont de fait de puissants vecteurs de syncrétisme.

Quand on s'accorda enfin sur le décès, Madan Olsen et quatre autres femmes s'appliquèrent à lui retirer ses vêtements, qui furent déchirés puis brûlés avec le lit sur lequel elle avait reposé jusqu'à sa fin. Métraux ne fait pas mention de cette pratique. Dans la région où j'ai enquêté, mais aussi dans le département de Nippes ${ }^{13}$, elle est partie intégrante du rite mortuaire quelle que soit l'appartenance religieuse du mort et de sa famille. Madan Olsen n'a à aucun moment questionné les gestes ou les actions des femmes s'activant à la toilette. Il semble qu'elle leur ait accordé l'autorité sur la procédure, et qu'elle était aussi satisfaite de voir « ces choses du diable (bagay dyab la) » partir en fumée. Les femmes lavèrent le corps à l'aide d'une eau dans laquelle baignaient diverses feuilles collectées dans le jardin attenant à la maison de Grann ${ }^{14}$. Rapidement séché, le corps fut ensuite vêtu, exclusivement de blanc - y compris les sous-vêtements. En Haïti, le blanc est aujourd'hui associé à la mort, au côté du noir ${ }^{15}$. Un morceau de

13. Obrillant Damus, com. pers., 2020.

14. Herskovits (2012 [1937], p. 207-208) et Métraux (1954, p. 291 et 1958, p. 219) font, tous deux, mention du fait que le cadavre est lavé avec une infusion de feuilles.

15. On ne trouve nulle indication de la couleur associée à la mort dans les travaux de Métraux. Herskovits, pour sa part, indique que le défunt est vêtu de noir et que les fournitures qui garnissent le cercueil sont aussi de couleur noire. Richman, 80 ans plus tard, évoque des 
tissu blanc fut attaché autour du visage de Grann et maintenait, de la sorte, sa bouche fermée. Les autres orifices, oreilles et narines, furent bouchés avec de l'ouate. Le gros orteil de gauche fut attaché à celui de droite afin que les pieds ne grandissent pas, me dit l'une des femmes. Herskovits (2012 [1937], p. 209) et Métraux (1954, p. 291; 1958, p. 219) indiquent également la mise en œuvre de ces procédés (mentonnière, gros orteil attaché et ouate) au cours de la toilette mortuaire, sans fournir plus d'explication. Herskovits avance cependant que la mentonnière doit permettre « de rendre possible l'identification d'un fantôme » et que la gorge du défunt ne s'assèche pas (2012 [1937], p. 209) et indique que les cotons placés dans les orifices sont le fait des paysans des mornes et que cette pratique n'a plus cours ailleurs. Le corps de Grann fut finalement recouvert d'un drap blanc. Celles qui avaient touché le cadavre se lavèrent les mains avec des feuilles d'oranger ${ }^{16}$, que l'on utilise également en d'autres occasions pour se laver de saletés prégnantes ou pour laver les viandes rouges. Des rèl se mirent alors à retentir. Une fois le corps apprêté, Olsen le conduisit dans une morgue aux Cayes. L'enterrement eut lieu une semaine après le décès de Grann. La date avait été fixée par Roseline, une des filles de Grann qui vivait à l'époque en Allemagne, et qui fut l'unique enfant, tous habitant à l'étranger, à faire le déplacement.

\section{La veillée}

Roseline arriva dans les mornes un jour avant l'enterrement, accompagnée de quatre des six enfants de sa sœur Rachelle ( $"$ Pasteur Alexis ${ }^{17} »$, Antoine, Stanley et Yves) et demi-frères, par leur mère, d'Olsen (Figure 1). Ils venaient de Port-au-Prince ou de République dominicaine. Tout comme Olsen, Pasteur Alexis, Antoine et Stanley (Yves est encore au lycée) ont fait carrière dans le secteur évangélique. Pasteur Alexis a fondé et dirige une église baptiste et un orphelinat à Port-au-Prince, il est aussi administrateur d'églises pour le compte d'une mission états-unienne. Antoine et Stanley, de leur côté, travaillaient

défunts revêtus de blanc (Richman 2018, p. 145). Faut-il en conclure qu'un changement est survenu au cours des dernières décennies, ou que des variantes régionales existent? Seule une enquête plus approfondie permettrait de le dire. On peut néanmoins d'ores et déjà avancer que la couleur n'est pas liée à la religion du défunt mais que le genre et les ressources engagées pour les funérailles expliquent en partie le recours à tel ou tel vêtement ou fourniture de telle ou telle couleur. En effet, dans les autres enterrements auxquels j'ai pu assister, les défunts étaient revêtus d'un costume noir et recouverts de blanc ou de noir tandis que les défuntes étaient vêtues et recouvertes (drap ou tulle) de blanc. Les personnes qui assistent aux enterrements, quand elles en ont les moyens, sont habillées plus souvent de blanc que de noir.

16. Métraux évoque des feuilles de citronnier (1954, p. 292).

17. Il n'est pas rare en Haïti que les hommes soient appelés par leur prénom précédé d'un titre lié à leur métier. C'est notamment l'usage pour les pasteurs. 


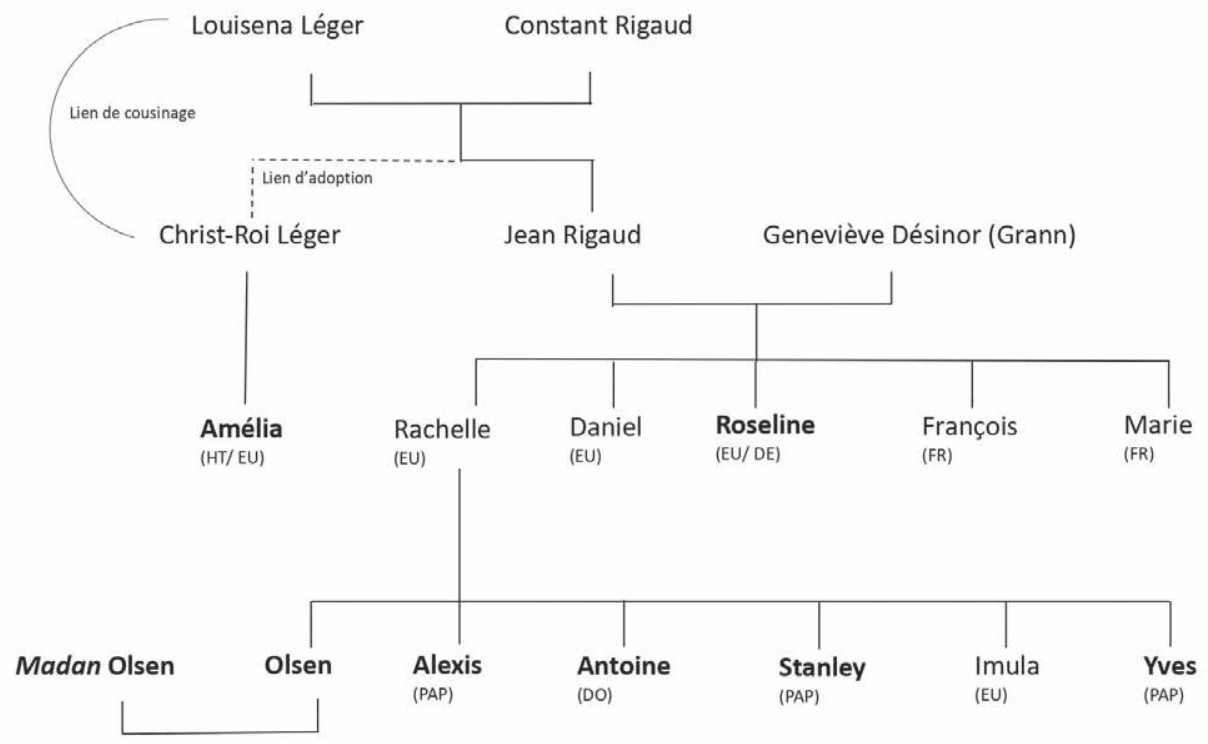

Fig. 1 - Relations de parenté entre les personnes mentionnées dans l'article (crédits : N. Mézié)

J'indique, pour les migrants, leur lieu de résidence et je signale, en gras, les personnes présentes aux funérailles. Légende $: \mathrm{DE}=$ Allemagne $; \mathrm{EU}=$ États-Unis $; \mathrm{FR}=$ France ; $\mathrm{HT}=$ Haïti $; \mathrm{PAP}=$ Port-au-Prince $; \mathrm{DO}=$ République dominicaine.

comme intermédiaires et accompagnateurs pour des missionnaires nord-américains. L'ascendant et le prestige de la famille qui réunit parents et descendants de Grann reposent, conjointement, sur la relative aisance économique de ses membres, la possession de terres (qui, au cours des dernières décennies, est allée néanmoins en s'effritant, à la suite de ventes), l'occupation de fonctions politiques, la ramification transnationale, et l'activisme et l'entreprenariat au sein du secteur évangélique.

Avec la veillée et, ensuite, l'enterrement, les leaders religieux de la famille prirent en main les funérailles et imprimèrent leur marque sur le déroulement et la teneur du rituel. Roseline décida de la tenue de la veillée le soir même de son arrivée. Des dizaines de chaises furent disposées dans la cour et à l'intérieur de la maison. Un générateur électrique fut loué pour alimenter des ampoules situées à l'intérieur et à l'extérieur. Café et thé furent servis à ceux qui s'étaient réunis.

À l'intérieur, dans la pièce centrale de la maison, s'entassèrent pasteurs et leaders d'églises alentours, aussi bien baptistes que pentecôtistes ou encore adventistes, et parents et voisins, tous protestants. Roseline fit une rapide 
apparition puis elle laissa à ses neveux le soin de diriger la veillée. S'enchaînèrent prières et cantiques, entrecoupés de rèl. Les rèl au cours des funérailles en milieu catholique et vodou retentissent avec intensité, du moins dans la petite paysannerie. Ils sont le fait de femmes qui peuvent être saisies de kriz, elle se jettent alors à terre, sont prises de secousse, tout en poussant des cris et en pleurant. Je n'ai observé aucun épisode de kriz au cours des funérailles de Grann. Plusieurs raisons peuvent être invoquées pour expliquer la relative rareté de rèl et cette absence de kriz: d'une part, rèl et kriz interviennent avec davantage de virulence quand la personne est décédée jeune ou de manière brutale, quand sa mort n'est pas pensée comme naturelle; d'autres part, les évangéliques baptistes, partageant cette sensibilité avec le protestantisme historique, valorisent une douleur publique contenue. Rodrigo C. Bulamah remarque également qu'au cours d'une cérémonie évangélique d'enterrement à Milot (nord d'Haïti), le pasteur réclamait des personnes présentes le contrôle de leurs émotions (2013, p. 123-124). La grammaire gestuelle et, plus largement, l'expression de la douleur qui sont valorisées en milieu évangélique baptiste renvoient à une certaine économie de la réserve et de la tempérance et, par conséquent, à une prise de distance revendiquée à l'égard du régime émotionnel en cours dans le vodou, mais aussi chez les pentecôtistes. Ces derniers, pour leur part, voient dans la maîtrise émotionnelle les signes d'une foi « froide (fredi, frèt) » ${ }^{18}$. À ces considérations religieuses, il faut aussi ajouter la question du statut social. En effet, dans l'élite urbaine catholique, il n'est pas convenable pour les femmes de s'épancher de manière aussi démonstrative.

À l'extérieur de la maison de Grann, sur la galerie et dans la cour qui fait face à la rue, une foule plus nombreuse était amassée, essentiellement des hommes qui ne se réclamaient pas du protestantisme. Ils jouaient au domino ${ }^{19}$, plaisantaient, dansaient, allaient et venaient entre la route et la cour, reprenaient parodiquement les cantiques qui leur parvenaient à travers les portes et les fenêtres. Ce jeu parodique n'est pas cantonné aux veillées. Au cours de combats de coq ou de petites sessions musicales sur la route en fin d'après-midi, il arrive que les participants, au milieu des rires, moquent leurs voisins protestants (pour leur soumission ou leur refus des plaisirs, par exemple) et dénoncent, frontalement ou non, les pasteurs et les missionnaires dans leur conquête des campagnes haïtiennes, qu'ils mèneraient, selon eux, en éradiquant la culture populaire vodou. Cette pratique, qui consiste conjointement à railler et accuser, à imiter et confronter, procède selon une modalité propre au carnaval et aux bandes

18. Sur le pentecôtisme comme « religion émotionnelle » et la formation au sein des églises de dispositions émotionnelle particulières, voir, par exemple, Fer (2005).

19. Le jeu de domino figurait parmi les nombreux divertissements de la veillée répertoriés par Métraux (1958, p. 222). 
$\operatorname{rara}^{20}$, dont les moqueurs et les imitateurs sont souvent de fervents adeptes. Ces voix de résistance à la conversion et à l'avancée protestante s'élèvent à mesure que les pratiquants du vodou et les fidèles catholiques, qui sont souvent les mêmes, deviennent minoritaires en certains endroits des mornes. Au cours des trente dernières années, les deux lieux-dits et leurs alentours immédiats où j'ai enquêté ont perdu la plupart de leurs ounfò (lieu de culte du vodou ayant à sa tête un hougan ou une mambo, respectivement spécialiste du rituel masculin et féminin) et de leurs gaguères où se déroulent les combats de coq (également proscrits par les courants protestants) ${ }^{21}$, tandis que plusieurs églises, des courants adventiste, baptiste et pentecôtiste, se sont implantées en bord de route, mais aussi dans des endroits plus isolés. Pour autant, on ne peut pas dire que la progression de ces protestantismes donne lieu à des conflits religieux. On assiste, plutôt, au quotidien, à une coexistence agitée, épisodiquement, de disputes théologiques (entre amis, compères, voisins, parents), de prosélytisme, plus ou moins frénétique, de la part des évangéliques et des pentecôtistes et de contre-discours railleurs des pratiquants du vodou. Dans les mornes, au jour le jour, prévalent les réseaux de dépendance, les associations de travail et les relations de parenté, qui transcendent le plus souvent les appartenances religieuses. Il est évident néanmoins que les fêtes et les cérémonies liées au cycle de la vie mettent en relief ce qui distingue les appartenances religieuses.

J'ai retrouvé une telle division, lors de la veillée, entre « intérieur protestant » et « extérieur catholico-vodou », dans d'autres funérailles évangéliques où j'ai pu observer des superpositions et des transitions, plus ou moins tendues, entre veillée d'intérieur et veillée d'extérieur. Au cours de l'une d'entre elles, la veillée évangélique d' " intérieur », soutenue par les seules voix des participants chantant et priant, s'était vue peu à peu assourdie par les bruits, les chants accompagnés de tambour et les rires de l'extérieur. À l'intérieur, où je me trouvais, l'irritation à l'égard de ceux du dehors qui ne « respectaient » pas le culte (« ils ne respectent pas ce qui est saint, ils ne respectent pas Jésus [yo pa konn respekte sa ki sen, y opa konn respekte Jezi] », dit une des femmes

20. Les bandes rara sont des bandes musicales à pied qui sillonnent les villes et les campagnes après le carnaval et jusqu'à Pâques. Sur ces bandes, voir le remarquable travail d'Elizabeth McAlister (2002) et sur le carnaval, voir celui plus récent de Rebecca Dirksen (2020).

21. J'ai recensé cinq ounfò et deux gaguères qui ont fermé, parfois en raison de la conversion du propriétaire, parfois sous la pression d'un pasteur et de ses fidèles, d'autres fois parce que les participants venaient à manquer, depuis l'implantation à Mòn Anvè de la première église évangélique. Ces fermetures ne peuvent pas pour autant être prises pour des indicateurs de la décrue de la pratique du vodou. En effet, le vodou domestique, forme commune du vodou rural, se pratique à l'écart des ounfò (Kerboull 1973). En outre, le vodou a toujours su se cacher pour mieux résister (en particulier au moment des campagnes anti-superstitieuses menées par l'Église catholique). Enfin, parmi les convertis au protestantisme évangélique et pentecôtiste, en dépit de l'anathème porté contre le vodou dans leur nouvelle communauté de foi, certains continuent à servir les lwa. 
présentes) a fait place au dépit; les cantiques ont cédé le pas aux clameurs de l'extérieur qui ont continué jusqu'en fin de nuit et chacun était parti, saluant ses connaissances au dehors. La cohabitation, parfois épineuse mais débouchant rarement sur des altercations, entre les deux espaces de veillée laisse percevoir les compromis auxquels peuvent se prêter les deux parties et l'impossibilité pour les évangéliques et pentecôtistes d'organiser des funérailles qui n'accorderaient pas une place à leurs parents non-convertis ${ }^{22}$. Il est difficile de refuser de distribuer à ces mêmes hôtes du kleren (rhum bon marché). Offert par la famille du défunt, le kleren coule habituellement à flot dans les veillées des funérailles vodou et catholiques. Si les invités ne devaient pas en recevoir, ils seraient prompts à protester et à taxer la famille d'avarice et d'irrespect. En début de veillée lors des funérailles de Grann, les hommes au-dehors ne se satisfirent pas du café et du thé, ils réclamèrent du kleren et l'obtinrent l'instant d'après. Mais sa consommation fut cantonnée à l'extérieur de la maison. L'alcool est prohibé par les églises évangéliques et pentecôtistes et le kleren, en particulier, fait l'objet des admonestations les plus sévères, parce qu'il est utilisé dans les rituels vodou et qu'il est incontournable dans les divertissements. Cependant, sa distribution constitue un des compromis ordinaires auxquels les évangéliques et les pentecôtistes se livrent pour maintenir leurs relations sociales avec des non-convertis. Lorsque, par exemple, les protestants font appel aux services d'une escouade (association de travail agricole) pour travailler leurs terres, ils doivent en retour fournir des journées de travail.

\section{Culte d'enterrement}

Le jour de la veillée et le lendemain, dès le petit jour, famille, amis et voisins s'activaient, pour les préparatifs de l'enterrement, dans la cour de Madan Olsen, boulangère et commerçante de détails et de demi-gros. C'est une femme à poigne qui jouit d'un réseau social ample. Elle dictait et supervisait le travail des uns et des autres. La cour de sa maison avait été transformée en quartier général pour les funérailles; là convergeaient les biens, les aliments, l'eau, la main d'œuvre faite des alliés et des professionnels (employés de la morgue, du loueur de chaises et tables, etc.). Quelques jours avant les funérailles, Madan Olsen fit laver de fond en comble sa maison. C'est chez elle que les invités de marque et les parents lointains se rendirent pour se restaurer, changer de vêtements, se reposer. La réalisation des tâches n'alla pas sans accrocs et disputes. On se plaignait de

22. Joël Noret (2004, p. 145) évoque, pour le Sud-Bénin, un « ordre négocié » des funérailles qui s'établit soit avec le retrait d'un des segments de la parenté (qu'il s'agisse des évangéliques ou des partisans des rites lignagers), soit avec la « juxtaposition » et la division des tâches (par exemple, tenue d'une veillée évangélique et organisation le lendemain des cérémonies funéraires traditionnelles). Voir également Katrin Langewiesche (2003, p. 243 304) sur les funérailles d'un chef catholique et les négociations autour du rituel à suivre. 
ne pas avoir reçu assez de sucre pour faire le café, on s'écharpa sur la quantité de nourriture à donner le jour de l'enterrement, mais c'est essentiellement le manque d'eau qui préoccupa Madan Olsen. Les citernes privées étaient vides depuis plusieurs semaines à cause d'une sévère sécheresse qui frappait la région. Le matin de la veillée, une distribution d'eau était orchestrée par l'ONG qui gérait deux citernes. Madan Olsen obtint de l'agronome en charge de la distribution - il est un habitué de la maison - des seaux à l'ouverture des robinets. Le jour de l'enterrement, Madan Olsen envoya une partie de sa main d'œuvre dans l'arrière-cour de Grann pour les préparatifs. Olsen, ses demifrères et Roseline rejoignirent la maison de Grann, également au petit matin. Pasteur Alexis s'attela à la programmation du service religieux, il circulait la Bible sous le bras et un cahier à la main et attribuait aux leaders religieux déjà présents l'office qui devait leur revenir. Olsen s'assurait, quant à lui, de la bonne exécution des derniers travaux à réaliser sur le tombeau. Roseline était happée par les personnes qui ne l'avaient pas vue depuis de nombreuses années.

De la ville des Cayes, vers 9 h 00, arriva le cercueil de blanc laqué, recouvert d'un capiton de blanc brillant. Grann y reposait en robe blanche. Le cercueil et son apparat (couronnes, gerbes de fleurs artificielles et tissus brillants aux teintes pastels) furent installés, selon les indications d'Olsen, dans le temple de la mission baptiste à laquelle Grann avait vendu ses terres 15 ans auparavant - les missionnaires américains se trouvaient alors en Floride. Tout comme au Ghana, l'exposition du corps est « un des points culminants » des funérailles en Haïti (De Witte 2003, p. 544). Moins d'une heure plus tard cependant, alors que la cérémonie n'avait pas encore commencé et que le temple était encore vide, le cercueil fut retiré du temple, à la surprise générale, comme nous le montrerons plus loin, et placé dans la cour de la maison de Grann où le culte prit finalement place. La foule, près de cent cinquante personnes, s'en vint alors dans la cour. Une fanfare avait été embauchée pour jouer au début et à la fin du culte. L'emploi d'un photographe-vidéaste, chargé de produire des images que Roseline voulait envoyer à ses frères et sœurs, à ses neveux et nièces, restés dans leur pays respectif, constituait une marque supplémentaire de prestige.

Chacun des participants se dirigea, avant de prendre place, vers le cercueil ouvert, qui fut fermé avant le début du culte. Les petits-enfants donnaient le programme de la cérémonie à ceux qui venaient exprimer leurs condoléances. Les membres de la famille prirent place sur les chaises qui avaient été installées des deux côtés de la cour. Une bâche apportait de l'ombre. Les hommes étaient vêtus de noir ou de beige et les femmes de couleurs claires, parfois avec une pièce en noir (la jupe ou une veste par exemple), à l'exception de Roseline qui était entièrement vêtue de noir avec une pièce de tissu en dentelle qui recouvrait ses cheveux. Les femmes de la famille s'étaient apprêtées avec soin, portaient des lunettes de soleil et avaient les cheveux impeccablement coiffés ; les arrières-petits-enfants de Grann étaient également vêtus d'habits pimpants 
neufs. Comme l'avait déjà remarqué Francis Affergan, pour la Martinique, l'enterrement est «l'occasion de montrer le costume et "d'envoyer de belles paroles" » (1975, p. 86). Seuls les petits paysans et les personnes sans le sou étaient habillés comme à l'accoutumée.

Au cours du culte, dirigé par Pasteur Alexis, se succédèrent prières, lecture de la Bible, cantiques et hommages grandiloquents à la défunte ${ }^{23}$, prononcés par des parents, qui tous l'érigèrent en chrétienne exemplaire. Aucun rèl ne se fit entendre. Une atmosphère solennelle régnait, accentuée par le fait que plusieurs orateurs s'exprimèrent en français. Une heure plus tard, le cercueil, porté par les petits-enfants, fut porté jusqu'au tombeau construit dans l'arrière-cour de la maison, à l'intérieur d'une petite bâtisse qui faisait office d'entrepôt. En milieu rural, et parfois même dans les bourgades où l'on peut trouver aussi un cimetière central, on érige les tombes pour les membres de la parenté décédés sur un terrain du lakou ${ }^{24}$, qui devenait alors le cimetière familial. Déjà du temps de la colonisation française au XVIII ${ }^{\mathrm{e}}$ siècle, les cimetières centraux ne recevaient pas tous les morts, il existait dans les plantations et les quartiers des cimetières particuliers (Debien 1974). Dans le nord du pays, à Cap-Haïtien et les alentours comme à Milot et à Samson, où il est expressément interdit d'enterrer sur ses terres ou dans le lakou, certaines personnes, qui se sont enrichies hors du pays, parviennent néanmoins à contourner la règlementation et à faire édifier une tombe sur leurs propres terres ou celles de leur famille ${ }^{25}$. Avec la recomposition du lakou et le morcellement des terres, d'anciens tombeaux se sont trouvés sur des terres qui n'appartenaient plus à la famille des défunts. Il arrive aussi que le cercueil soit placé dans un tombeau sur des terres qui autrefois appartenaient au lakou et qui sont à présent éloignées de la maison du défunt. Mais, ce qui semble le plus courant aujourd'hui dans les mornes, c'est de construire un tombeau dans la cour de la maison où logeait celui qui est mort. Pour éviter aux morts une « disgrâce éternelle » (Richman 2018, p. 124), le cercueil n'est le plus souvent pas mis en terre mais une place lui est aménagée, hors-sol, dans le tombeau. Les tombeaux sont faits de maçonnerie et peints de couleurs variées, une croix de maçonnerie ou de fer peut y être élevée, parfois d'autres ouvrages de fer sont ajoutés. On voit de nos jours, de plus en plus, des couronnes agrémentées de fleurs de plastique et de rubans brillants. Il arrive que le bâti soit réalisé alors que la personne est encore vivante et selon ses instructions;

23. Le moindre événement en Haïti (comme l'ouverture d'une boulangerie, par exemple) est accompagné, suivi ou précédé de discours où des hommes, principalement, font montre de leur talent oratoire et sont jugés en conséquence.

24. Le lakou a pendant longtemps été la structuration spatiale de la famille élargie en milieu rural: les enfants construisant leurs maisons auprès de celle de leurs parents, auxquelles parfois venaient se greffer d'autres membres de la parenté (Bastien 1985 [1951], p. 43-53; Laguerre 1978).

25. Rodrigo C. Bulamah, com. pers., 2020. 
ainsi en avait-il été pour Grann ${ }^{26}$. Ces tombes atteignent parfois de si grandes proportions qu'elles paraissent avoir absorbé plus d'attention et d'argent que la construction de la maison. On rencontre des usages semblables en Jamaïque où les tombes, fréquemment érigées sur les terres familiales, sont conçues comme de petites maisons qui abritent les morts (Horst 2004). Le coût, dispendieux, doit être supporté par les enfants, qui se prêteraient au déshonneur s’ils devaient manquer à ce devoir. La tombe constitue ainsi un signe de richesse et une marque de prestige pour la famille. Cependant, quand le défunt est trop jeune, ou bien âgé et délaissé par ses parents, ou encore la famille trop pauvre ou le conjoint peu fortuné, il peut arriver que le cercueil soit mis en terre et recouvert d'un simple pavement de ciment. Cette pratique semble se répandre, en raison de l'appauvrissement de la paysannerie des mornes et sous l'influence d'un protestantisme évangélique plus récalcitrant aux dépenses somptuaires. À l'opposé, certains rares tombeaux nouvellement construits exhibent une majesté et un décorum qui sont souvent rendus possibles grâce à l'argent de la diaspora.

Le tombeau de Grann était en maçonnerie d'une hauteur d'un mètre cinquante. Une croix le surplombait, mentionnant la date de son décès. Sans être somptueux, il n'était pas non plus celui du commun des mornes. Dans les environs, ce genre de tombe est l'attribut de la paysannerie aisée. On s'y pressa pour rendre les derniers hommages tandis que la fanfare jouait encore. Pour finir, les participants reçurent un plat composé de riz, d'une sauce de pois noir et de morceaux de viande et un verre de boisson gazeuse. Au fil de l'après-midi, les personnes quittèrent la maison de Grann.

Culte, mise au tombeau et distribution d'un repas aux invités viennent achever les devoirs évangéliques envers le mort en Haïti. Il est ensuite laissé au soin de la seule mémoire des vivants. En respect avec la tradition théologique protestante, on ne doit lui accorder ni culte, ni l'élever au rang d'ancêtre ${ }^{27}$. La différence est donc notable, d'une part, avec la manière dont le rituel catholico-vodou poursuit, à l'issue de l'enterrement, son travail et son action sur le défunt, et en

26. Bastien remarque que cette « coutume » était encore à l'œuvre dans le nord-ouest d'Haïti - ses enquêtes de terrain se déroulèrent de 1940 à 1944 (1985 [1951], p. 141). Richman, pour sa part, suggère que ceux qui peuvent se permettre aujourd'hui de construire leur tombe de leur vivant sont ceux qui ont accès à des ressources provenant de parents ayant migré (2018 [2005], p. 68).

27. Les réformateurs protestants se détournent de l'au-delà et du sort de l'âme et prônent la sobriété pour les funérailles (Crousaz 2013; Grosse 2019). Mais comme le constate Christian Grosse, très vite « émancipé de l'emprise de l'Église, le rite funéraire va donner progressivement prise aux besoins de distinction sociale » (Grosse 2019, p. 191). Il faut aussi compter avec les divers courants protestants qui ont proliféré après la Réforme et ont apporté leur lot de transformations, tout comme avec les compromis, adaptations et métissages qui se sont développés à mesure que le protestantisme évangélique, pentecôtiste et néo-pentecôtiste s’est globalisé (Mullins 1990; Noret 2004; Rus 2014). 
particulier sur son âme ${ }^{28}$, et, d'autre part, avec la manière dont est conçue l'eritaj (héritage). L'eritaj, « construction socio-symbolique la plus importante de la culture paysanne haïtienne » (Lowenthal 1987, p. 194), renvoie à un groupe de cognats liés à un ancêtre apical, qui possèdent en commun: des $l w a$, un répertoire de rituels, des terres et des relations, ainsi que des obligations cultuelles à l'égard des esprits des parents défunts (ibid., p. 194-211; Dalmaso 2018). Ces obligations cultuelles consistent principalement en offrandes annuelles aux ancêtres et lwa de la famille. Les convertis haïtiens au protestantisme évangélique et pentecôtiste refusent de participer aux rites familiaux pour les esprits des ancêtres et d'entretenir le moindre commerce avec les morts. Les relations entre les vivants et les morts, actées et médiatisées par l'institution religieuse et par le rituel dans d'autres religions, sont coupées. Le calendrier rituel post-mortem est réduit au seul culte d'enterrement, qui endosse, de manière concentrée, l'expression collective du deuil. On assiste ainsi, tout comme au Bénin, à un «basculement de la place des morts » (Noret 2004, p. 150) dans la mesure où « toute construction d'une mémoire religieuse des défunts est proscrite » (ibid., p. 153) et que « tout geste (ou toute parole) cultuel à l'attention des défunts est désormais considéré au mieux comme sans objet, au pire comme satanique » (ibid., p. 150).

\section{Une famille transnationale : maintien et distension des liens}

Comme il a été dit, tous les enfants de Grann ont quitté le pays, les deux plus âgés pour la France, les autres pour les États-Unis. Cette configuration transnationale de la famille est commune aujourd'hui en Haïti, et plus globalement dans la Caraïbe. Terre d'accueil dans les décennies d'après indépendance (Fouron 2020), la nation haïtienne va ensuite « à travers l'émigration, s'étendre au-delà de ses frontières géographiques » (Laguerre 1998, p. 13). Au début du $\mathrm{Xx}^{\mathrm{e}}$ siècle, des dizaines de milliers d'Haïtiens quittent leur île pour être embauchés dans les plantations de canne à sucre à Cuba (Casey 2017) ou en République dominicaine. Avec la dictature des Duvalier (1957-1986), émigrent des familles de la bourgeoisie, des classes moyennes et de la paysannerie aisée. C'est au moment de la fin du régime Duvalier père que les enfants de Grann migrèrent, en partie grâce à la vente de terre, à l'exception de la benjamine qui partit au milieu des années 1980. Les États-Unis, le Canada et la France constituaient alors le trio de tête des pays de destination. Au cours des décennies suivantes, alors que s'enchaînent les crises politiques et économiques, les migrations

28. Parmi les actions rituelles qui se déroulent à l'issue de l'enterrement citons la neuvaine (Herskovits 2012 [1937], p. 212-213), les dernières prières, le dessounin, le port du deuil, la cérémonie du wete mò nan dlo (Métraux 1958, p. 228-234; Richman 2018, p. 124-128), la fête des morts et les offrandes qui leur sont faites (Métraux 1958, p. 234-235; Smith 2010). 
s'intensifient ${ }^{29}$. Dans les années 2000 , on comptait, très approximativement, un million d'Haïtiens vivant à l'étranger (Bidegain 2013).

Roseline, au moment du décès de Grann, travaillait pour les forces armées des États-Unis en tant que responsable des achats pour la restauration des bases militaires en Allemagne. Elle vivait ainsi dans ce pays depuis plusieurs années, où elle s'était convertie au bahaïsme, après avoir été en activité sur d'autres bases militaires à travers le monde ${ }^{30}$. Sans enfants, sans mari, jouissant d'une certaine aisance financière par son emploi, elle est devenue une mère substitutive pour plusieurs des enfants de sa sœur Rachelle, dont on disait, sans trop savoir, que fragile psychologiquement, elle avait sombré dans la démence aux États-Unis. On pourrait se demander si son célibat n'est pas stratégique, tout comme il peut l'être pour d'autres raisons dans la paysannerie béarnaise (Bourdieu 2002), dans le sens où il semble viser à soutenir la migration des membres de la famille, en prenant à sa charge les enfants de sa sœur. Roseline est celle qui concentre le « capital migratoire» (Laurent 2018) de la « famille à distance » et qu'elle se doit de faire circuler en œuvrant à faire sortir d'Haïti ses parents consanguins. Détentrice d'un passeport états-unien, parlant parfaitement l'anglais, familiarisée, par son travail, avec l'administration américaine, elle a obtenu qu'Imula, la benjamine de la fratrie, s'installe aux États-Unis ${ }^{31}$, et qu'Antoine la rejoigne en Allemagne. Roseline, « transmigrante ${ }^{32}$ » (Schiller 1999), est une pièce charnière, une intermédiaire et un élément-liant dans la constitution et l'entretien de la « famille transnationale ». Elle travaille au maintien des liens entre les différentes parties à travers le monde et investit temps, argent et attention à cette fonction. Le versant atlantique américain de la famille entretient avec une certaine fréquence leurs relations. Roseline participe, quand elle le peut, aux

29. Le profil socio-économique des migrants évolue, si les migrants formés continuent à fuir le pays, on trouve de plus en plus d'individus de milieux modestes et pauvres à partir, tandis que les pays de migration, en raison de la fermeture des frontières et des politiques sécuritaires au Nord, s'étendent à l'Amérique centrale et latine et aux Caraïbes (Audebert 2017).

30. Je tiens ces informations sur Roseline et ses frères et sœurs, d'Olsen, d'Antoine et de pasteur Alexis. Je n'ai rencontré Roseline qu'une seule fois, lors des funérailles de Grann. Nous avions alors très peu échangé, les circonstances ne prêtant pas à de longues discussions et encore moins à un entretien. Elle n'avait d'ailleurs montré ni surprise ni intérêt à ma présence. Je suppose que mon jeune âge (j'avais alors 25 ans), mon allure « dépenaillée », et mon « association » avec Madan Olsen expliquent en partie cette indifférence. Roseline possédait un réseau social déjà bien ancré en «pays étranger (peyi blan) » et sa position sociale n'avait rien à envier à nombre de Blancs. Elle n'avait aucune raison de chercher à tout prix à établir un lien avec moi.

31. Celle-ci mourut en 2006, emportée par une maladie.

32. «Les transmigrants sont des personnes qui se réclament et sont revendiquées par deux, ou plusieurs, Etats-nations au sein desquels elles sont incorporées en tant qu'acteurs sociaux, l'un de ces États étant connu comme l'État d'origine » (Schiller 1999, p. 96; la traduction est la mienne). 
rituels qui marquent le cycle de vie (mariage, enterrement) et fait alors connaissance avec les récents entrants dans la parenté (petits amis, fiancés, enfants en bas-âge, filleuls, etc.). Elle circule ainsi, pour la famille, entre différents États des États-Unis, en République dominicaine et en Haïti. Comme dans d'autres contextes transnationaux caribéens (Horst et Miller 2005 ; Plaza et Plaza 2019), les relations avec les parents vivant au loin sont préservées, au quotidien, grâce aux appels passés avec des téléphones portables qui ont pris le relais des appels réalisés depuis des phone call centers et des cassettes letters que s'envoyaient Haïtiens de l'étranger et Haïtiens restés au pays dans les années 1980-1990 (Richman 2018 [2005], p. 212-249). Au cours des années 2010, se sont imposés Whatsapp et Facebook. Sur ce dernier réseau social, Roseline, que je suis, est particulièrement active, elle répond en anglais ou en français, usant toujours de termes de parenté pour saluer et louer les uns et les autres, aussi bien ceux du versant atlantique européen que ceux du versant américain. Le recours à un photographe-vidéaste lors de l'enterrement de Grann, nous l'avons dit, avait pour but de pouvoir disposer d'images que Roseline allait faire circuler au sein de la « famille transnationale ».

Karen Fog Olwig souligne que les « mariages et funérailles sont d'une importance cruciale dans les réseaux familiaux de migrants parce qu'ils constituent des événements où les parents qui vivent dispersés peuvent se rencontrer et valider leur parenté partagée et leurs origines communes » (2002, p. 205; voir aussi 2009; la traduction est la mienne). Dans le cas de funérailles, on attend des enfants du défunt établis à la ville, et plus encore à l'étranger, de pourvoir aux dépenses engagées pour la morgue, la cérémonie et autres nécessités. Les transformations que connaissent les funérailles en Afrique et dans la Caraïbe sont, en partie, liées aux «vies transnationales »: « les migrants à l'étranger jouent un rôle important dans l'organisation, le financement et la tenue des cérémonies » (Mazzucato, Kabki et Smith 2006, p. 1048; la traduction est la mienne). Les dépenses considérables consenties pour réaliser des funérailles avaient déjà été notées par Métraux (1954, p. 19), Bastien (1985 [1951], p. 141) et Murray (1977). Ce dernier explique que la circulation et la vente des terres est due, en grande partie, aux coûts à assumer pour des funérailles ponctuées de rituels onéreux. L'argent provenant des parents migrants apporte une nouvelle source de financement qui permet d'offrir des funérailles, si ce n'est fastueuses, du moins opulentes, et contribue à alimenter une «véritable "industrie funéraire" » (Noret 2014, p. 56). Karen Richman décrit cette évolution à Léogane, où maisons ${ }^{33}$ et tombes gagnent en opulence grâce aux envois de fond et aux dépenses des membres de la diaspora (2018 [2005], p. 68). Handerson Joseph fait le même constat à Fonds-des-Nègres et Pemerle, deux localités du sud du pays (2015, p. 187-188 et 2020). Les funérailles de Grann avaient peu à voir

33. Sur les kay dyaspora (maisons diaspora) haïtiennes, voir Joseph 2020. 
avec les funérailles que l'on voit habituellement chez les petits paysans des mornes: fanfare et photographe-vidéaste sont mobilisés davantage en milieu urbain ou dans les grosses bourgades, la pompe du cercueil ne ressemblait en rien aux cercueils de bois exécutés sommairement par un ébéniste local. Le coût de la construction du tombeau, du séjour à la morgue, ainsi que de la cérémonie ont été pris en charge par Roseline ${ }^{34}$. Au moindre coût ou achat supplémentaire (de sucre, de kleren, de poudre de café, etc.), Madan Olsen en avertissait Roseline qui tenait en mains, tout au long des funérailles, un cahier où elle annotait les dépenses et le nom de ceux qui s'activaient pour la réussite de la cérémonie. Elle a ensuite chargé Olsen, à qui elle avait remis de l'argent liquide, de rembourser les frais avancés par ces personnes.

Quand il est question de diaspora, les chercheurs portent davantage leur attention, d'une part, sur ces figures agrégatives, qui gèrent les relations au sein de la parentèle et, d'autre part, sur ce que Fog Olwig appelle la « construction d'un locus familial (home making) » (2002); ils s'intéressent moins aux relations en pointillés ou à celles qui se défont, au « détachement » (Candea et al. 2015), aux interruptions, aux ruptures, aux « perdus de vue ». À l'inverse de Roseline, les deux enfants naturalisés français de Grann font partie de ces personnes qui rompent « physiquement », volontairement ou non, dans la migration, sur le très long terme et parfois de manière définitive, avec leur pays d'origine. Le frère et la sœur aînés ont migré au début des années 1980 et ne sont depuis jamais rentrés en Haïti. Le premier est devenu mécanicien automobile et la seconde a connu de longues périodes de chômage. Ils n'ont donc pas réussi économiquement à l'égal de leur sœur Roseline, et ils n'envoient pas, à ce que j'ai pu savoir, de fonds en Haïti. Leur migration s'est accompagnée d'une distension des liens avec leurs parents, à l'exception de Roseline, en Haïti et aux États-Unis. Tout indique que leur migration conjointe a réduit leur cercle familial aux deux familles installées en banlieue parisienne et que les difficultés économiques ont limité les relations avec le reste de leurs parents haïtiens. Olsen et ses demi-frères savaient peu de choses de leur oncle et tante français jusqu'à ce que Facebook établisse une forme de communication et d'interconnaissance. Au cours des funérailles, je n'ai pas entendu leur nom prononcé, ils n'ont pas été mentionnés dans les discours, comme s'ils s'étaient évanouis de la mémoire des habitants de la localité et que leurs proches ne voulaient pas la réactiver. Quelques vieilles personnes des mornes demandaient des nouvelles de Rachelle, certains la confondaient avec Roseline et personne ne les corrigeait. On ne savait plus qui était qui, ni qui était vivant, ni qui était mort. Il y avait une femme, en revanche, dont on savait très bien qui elle était, parce qu'une fois l'an, elle venait, dans la précipitation, visiter son père Pasteur Christ-Roi, ami

34. Je n'ai pas pu savoir si son frère et sa sœur résidant en France y avaient participé. Il est vraisemblable que non. 
de toujours de Grann: Amélia. Amélia, qui revendique une relation de parenté avec Grann, allait au cours de l'enterrement, comme nous allons le voir, faire place, donner les places, se donner une place. Tenter de dévoiler les intentions circonstancielles de ses actions et décrypter les réactions et les interprétations des gens des mornes à sa présence et à ses paroles nous permettra de comprendre le coup de théâtre que fut l'événement que nous allons à présent relater: le retrait du cercueil du temple EBM et son exposition dans la cour de la défunte.

\section{Frontières de la parenté et concurrence entre églises}

Alors que le cercueil avait été placé par les employés de la morgue dans le temple de la mission EBM, Roseline prit la décision, en concertation avec Amélia et sans s'en entretenir avec ses neveux et le reste de la famille, d'y retirer le cercueil et de le relocaliser sur le devant de la galerie de la maison de Grann. Au début du culte d'enterrement, Roseline, pour dissiper les malentendus et éviter la propagation de commérages, mit sur le compte des caprices d'une vieille femme et de la tradition le choix de l'emplacement du culte:

Et puis excusez-moi parce que j'ai changé la place. Je n'ai rien contre le pasteur ou bien l'église mais comme vous savez, une personne âgée haïtienne, si elle te dit quelque chose avant de mourir. [...] Alors je suis obligée de faire ce qu'elle désirait. [...] Elle avait dit c'est l'église de Christ-Roi où j'irai ou bien ici. (Roseline, discours en créole)

Amélia, mariée à un militaire à la retraite états-unien, est née à quelques kilomètres de la maison de Grann. Son père, âgé de 83 ans en 2007, était un évangéliste de la première heure : il avait sillonné à pied la Grand'Anse et implanté plusieurs dizaines de petites églises, après s'être converti auprès d'un missionnaire cubain qu'il avait rencontré dans la ville de Jérémie en 1940. Un temps baptiste, il rejoignit le pentecôtisme alors que s'affirmait chez lui un don de guérison et de parler en langues. Pasteur Christ-Roi était un ami de longue date de Grann. Il avait été élevé par les parents du mari de Grann, Jean Rigaud: Louisena Léger, une lointaine cousine de Christ-Roi, et Constant Rigaud. Amélia a suivi son père dans la carrière protestante, en investissant dans le pentecôtisme historique. Dans la ville de Jérémie, où elle vit une partie de l'année, elle a érigé un temple pentecôtiste, construit un orphelinat et une école.

Amélia arriva en voiture aux funérailles. Elle se joignit immédiatement à Roseline, dont le visage était marqué par le chagrin et qui semblait légèrement déboussolée. Toutes les deux sont grandes, sèches de corpulence et dans leurs gestes. L'une était vêtue de beige, l'autre de noir. Elles formaient un couple remarquable et d'autant plus remarqué que leur présence se donnait sur le fond de leur longue absence. Aux yeux de tous, elles incarnaient une figure de la réussite dans un pays où les aspirations du plus grand nombre est de « chercher 
la vie (chache lavi) » à l'étranger et où les États-Unis et l'Allemagne sont les plus hauts placés dans la hiérarchie locale des pays de migration ${ }^{35}$. Leur alliance dans la direction de l'enterrement se fondit sur cette identité diasporique et sur l'image de réussite qu'elles projetaient. Amélia, son appareil photo à la main, s'activa dès son arrivée. Après que le cercueil fut déplacé dans la cour de Grann, elle s'occupa de déterminer les places des uns et des autres:

Vous pouvez laisser ces rangées-là pour la famille (elle pointe du doigt trois rangées). Toutes les autres chaises sont free. Tu peux t'asseoir là (elle s'adresse à une personne qui s'avance), c'est pour les frères, les sœurs... Vous entendez? [...] Cet enfant-là (elle pointe du doigt vers un enfant assis), il est de notre maison $\left(\right.$ lakay nou $\left.{ }^{36}\right)$. Les gens qui ne sont pas de notre maison, les gens qui sont en-dehors de la famille, ils peuvent s'asseoir ici. Là, c'est les frères, les neveux, les nièces, c'est la famille. (Amélia, en créole, peu avant le culte)

En attribuant les chaises, elle déterminait un en-dedans de la famille, dans lequel elle s'incluait (lakay nou), et un en-dehors de la famille et elle témoignait de sa connaissance des composantes familiales de la localité et des environs. Une « expertise » qu'elle donnait aussi à entendre lors du culte, en prenant à son compte le discours, habituel en pareille circonstance, des frontières et de l'identité de la parenté ${ }^{37}$ du défunt. Au cours de ce discours, elle allait tirer la couverture de la généalogie vers son père, et par conséquent elle-même, et louer le travail évangélisateur de ce dernier.

Mon papa a été élevé chez Constant Rigaud, mon papa m'a toujours dit que c'est Constant qui a acheté le syllabaire, qui donnait des leçons à Jean. Aujourd'hui si mon papa est pasteur, il sait lire, c'est grâce à Constant Rigaud. [...] Constant, c'est le grand-père de ces mesdames qui sont assises là. Il est resté avec cette petite malheureuse qui s'appelait Louisena Léger qui était la cousine de mon papa. Les relations de mon papa avec Louisena c'était comme s'il était rentré dans la famille. [...] Moi-même, je suis de la famille de Geneviève, moi je suis de la famille Geneviève. (Amélia, discours en créole)

La revendication de filiation avec Grann à laquelle procédait Amélia n'avait en soi rien d'aberrant. En Haïti, la circulation des enfants entre familles et l'adoption d'enfants, d'un parent ou non, non déclarée à l'État, sont très courants.

35. Sur la hiérarchisation des pays de migration, en fonction des conditions de vie, de la valeur de la monnaie nationale, des possibilités d'emploi et de mobilité sociale, voir Joseph (2015).

36. $\mathrm{Si}$ « nou » en créole haïtien peut vouloir dire « vous » et « nous », il renvoie ici sans ambiguïté au pronom « nous ».

37. J'emploie à dessein "frontières de la parenté » pour dire combien les relations d'alliance, de consanguinité et d'adoption sont pensées conjointement avec les lieux d'origine et d'installation. La proximité spatiale entre des individus, non apparentés biologiquement, peut créer de la parenté. 
Les frontières de la famille (fanmi a) ne sont pas établies par les seuls liens de sang, elles peuvent donc se dilater pour intégrer ces enfants, tout comme peuvent être inclus d'autres individus à l'exemple de voisins ou encore de parents rituels ${ }^{38}$. La narration généalogique et l'insistance sur sa filiation avec Grann lui servirent de légitimation à son association avec Roseline pour mener les funérailles.

Amélia avait toutes les raisons de ne pas vouloir que le culte d'enterrement ait lieu dans le temple de la mission EBM et ses raisons ne sont pas théologiques. L'entreprise pentecôtiste d'Amélia était en concurrence avec la mission EBM. Cette rivalité, qui n'a jamais atteint le stade du conflit déclaré, doit être considérée à l'aune de l'expansion dans le paysage religieux haïtien du protestantisme, sous ses formes les plus diverses. Au cours de l'occupation américaine (1915-1934), le protestantisme évangélique et pentecôtiste a gagné en visibilité en Haïti. Ces courants protestants, à partir des années 1980, ont connu une progression spectaculaire - qui s'est d'ailleurs encore accrue après le séisme de $2010^{39}$. Le pentecôtisme a rapidement gagné en force et supplanté le courant baptiste tandis que l'église catholique voyait en son sein croître le mouvement charismatique fortement influencé par le pentecôtisme. D'autres courants et mouvements sont venus enrichir le paysage religieux haïtien, à l'exemple du néo-pentecôtisme, parfois aussi qualifié de charismatisme troisième vague, qui prêche la théologie de la prospérité et la " guerre spirituelle » (que l'on retrouve essentiellement à Port-au-Prince et dans les grandes villes), des Témoins de Jéhovah, des Mormons, des Adventistes, de l'Armée céleste. En 1960, on comptait près de $13 \%$ de protestants (Larose 1994, p. 121). En 2003, d'après le dernier recensement effectué en Haïti, on estimait que $30 \%$ de la population nationale se revendiquait protestante dont $8 \%$ aurait été pentecôtiste (Clorméus 2014, p. 161). Mais dès 1997, des chercheurs évoquaient le chiffre de $40 \%$ de protestants dans la capitale (ibid., p. 162). Il est certain qu'au cours de ces dernières années, ces chiffres ont encore augmenté. Les missionnaires nord-américains ne sont pas étrangers à ce succès. Cependant, l'on sait aussi que

38. Il n'est pas possible, dans les limites imparties de l'article, de discuter des catégories endogènes de la parenté dans la paysannerie haïtienne (comme celles de "ras », "lakay », " san », "fanmi », etc.). Sur cette question, voir par exemple: Woodson 1990, p. 209-266; Lowenthal 1987, p. 185-194; Laguerre 1978.

39. Parmi les premiers travaux sur le protestantisme en Haïti, on peut citer Conway (1978) et Romain (2004 [1986]). Ce n'est qu'au tournant des années 2000 que des anthropologues, dont plusieurs avaient d'abord étudié le vodou, se penchent sur les bouleversements dans le paysage religieux haïtien et dans celui de la diaspora haïtienne avec la montée en flèche des conversions et de l'implantation de missions et d'églises évangéliques, pentecôtistes et néo-pentecôtistes, sans compter l'arrivée de nouveaux mouvements religieux. Voir entre autres: Louis 2015; Brodwin 2003; Corten 2001; Hurbon 2001; McAlister 2013; Mézié 2016; Richman 2012. 
la force du protestantisme, au contraire du catholicisme, est aussi de s'indigéniser très rapidement, comme le rappelle Serge Larose (ibid.). Dans les quartiers, les bourgades, mais aussi en certains endroits des campagnes, la prolifération des temples provoque des situations de concurrence aiguë et contribuent à la circulation de rumeurs sur l'usage de la sorcellerie par des pasteurs pour accroître le nombre de leurs ouailles et/ou se défaire de concurrents gênants ${ }^{40}$. Des chercheurs, souvent à partir de la perspective du choix rationnel et de l'analogie entre logiques de marché et logiques religieuses, ont relevé la compétition et les luttes engendrées par la pluralisation d'un champ religieux, qu'elles s'opèrent entre religions distinctes ou entre branches du christianisme ou encore entre dénominations et courants protestants (Woods 2012; Mayrargue 2009).

Au cours des années 2005-2007, à Mòn Anvè, la désaffection pour les petites églises aux alentours de la mission EBM en raison de l'attractivité de cette dernière constitue un motif d'inquiétude pour leurs pasteurs. La fragmentation, dynamique constitutive du protestantisme, a prospéré dans la localité jusqu'à la fin des années 1990. Les églises implantées entre 1980 et 1990 ont toutes connu au moins une scission, sous le couvert de différends théologiques ou de condamnation morale portée contre les leaders. Créer son Église offrait alors plusieurs avantages: l'apport d'un revenu (aussi faible fut-il) et le prestige dont tout pasteur-fondateur d'église peut se prévaloir sans compter l'assurance de pouvoir mobiliser les fidèles pour des fins personnelles (culture des terres, petits travaux, etc.). Par ailleurs, jusqu'au début des années 1990, la réserve de non-convertis était encore loin d'être épuisée et ne compromettait donc pas la dynamique en cours. Entre la fin des années 1990 et le début des années 2000, la mission EBM des blan multiplia ses actions d'évangélisation et ses activités de culte, accueillit une clinique annuelle, fonda une école, proposait un programme alimentaire et d'assistance aux plus pauvres... Ces biens spirituels et matériels prodigués conjointement contribuèrent à attirer de nouveaux fidèles ${ }^{41}$ et à placer la mission en situation de quasi-monopole dans la localité. Dans les mornes, les petites églises baptistes ou pentecôtistes, dirigées par des pasteurs vieillissants, peu formés et sans ressources extérieures, tentaient bon gré mal gré de résister avec les moyens du bord. L'Église pentecôtiste du

40. Un pasteur qui fonde une église et remplit rapidement son temple peut être accusé d'avoir pactisé avec le diable ou d'avoir recouru à un bokò (spécialiste du vodou qui travaille des « deux mains »). J'ai entendu dire également que des pasteurs avaient pu perdre leurs ouailles sous l'action d'un baka (être maléfique qui peut s'introduire dans les maisons, les bâtiments et conduire à la perte des biens du propriétaire). Tout comme il est possible de disqualifier un « concurrent » en insinuant qu'il n'a que partiellement abandonné le vodou.

41. Cette mobilité, souvent réversible (à la manière dont le sont les conversions), des chrétiens d'une Église à l'autre et d'une dénomination à l'autre n'est pas le propre de l'endroit. On la rencontre également en ville mais aussi, par exemple, et dans un tout autre contexte, dans l'Amazonie péruvienne chez les Yine (Opas 2019). 
père d'Amélia, dont il avait laissé la direction à un jeune pasteur entreprenant, était la seule église dans la localité qui ne souffrait pas de la perte de fidèles qui rejoignaient celle des missionnaires; mieux, elle gagnait, en 2005 et 2006, des fidèles. En 2006, Amélia construisit, sur la route qui mène à la localité, un Centre d'économie domestique. Elle envisageait, par ailleurs, de fonder un nouveau temple. Autrement dit, Amélia accroissait ses activités religieuses dans les mornes, sur le terrain des missionnaires de l'EBM, entrant ainsi dans une lutte de concurrence pour capter les fidèles et les nouveaux convertis ${ }^{42}$. Elle semblait vouloir faire contrepoids en investissant dans les mornes, sur « les terres de mon père (tè papa'm) », me dit-elle un jour. À Jérémie, chef-lieu du département de la Grand'Anse, elle avait su se bâtir un fief évangélique (église, école et orphelinat). Sans doute comptait-elle capitaliser sur cette réussite pour rivaliser avec le couple de Floride. On ne peut pas, en tous cas, évacuer les composantes du prestige et de la lutte d'influence pour comprendre tout à la fois le déplacement du cercueil et, plus largement, le rôle de premier plan joué par Amélia au cours de l'enterrement, perçu comme aberrant par certains membres de la famille comme nous allons le voir à présent.

\section{«Sous un arbre (Anba pyebwa) »: contestation des gens des mornes}

Cette décision, inattendue et non négociée avec la famille, suscita le mécontentement d'Olsen, de Madan Olsen et de sa mère, qui, il faut le rappeler, étaient des soutiens de la première heure de la mission. Olsen montra sa désapprobation; il était celui qui avait fait déposer le cercueil dans le temple EBM, là où lui et sa famille se rendaient au culte. Il alla faire état de son agacement à ses demi-frères auxquels il fit savoir qu'il était irrespectueux de changer de place le cercueil à la dernière minute, tant pour le travail des employés de la morgue, dont l'un d'eux s'était plaint de cette décision dans la cour d'Olsen, que pour les missionnaires qui mettaient à disposition leur église. Par ailleurs, le culte déplacé dans la cour de Grann entravait le travail des maçons qui terminaient le tombeau. Pasteur Alexis lui répondit qu'ils devaient éviter les diskisyon (« discussions »). Antoine ne voulut pas non plus polémiquer: « Et bien pour moi, ça ce n'est rien [...] pour moi ça ce n'est rien (e byen pou mwen sa pa anyen [...] pou mwen sa pa anyen) ». Du côté de Madan Olsen et de sa mère, les raisons de la colère étaient autres. Toutes deux blâmaient Amélia et attaquèrent sa personnalité : « cette personne n'est pas bonne (moun nan pa bon non) ». Par ne pas être « bonne », il faut entendre, en l'occurrence, qu'elle a l'habitude de se soustraire aux règles du jeu social (et, en particulier,

42. Au cours d'une excursion à Jérémie, j'ai séjourné chez Amélia. J'ai ainsi pu discuter avec elle de son « projet de développement » pour la région des mornes qui l'avait vu naître et pu prendre connaissance de son désir, qu'elle ne m'a pas caché, de faire face à Pasteur John. 
de celles de l'hospitalité comme nous allons le voir) et qu'elle ne satisfait pas aux exigences sociales liée à son statut. Ce déplacement du cercueil souleva aussi l'incompréhension de beaucoup d'autres parents des mornes. Au-delà du retrait du cercueil de l'église, c'est le rôle de premier plan que s'accorda Amélia qui nourrit leur énervement.

Après l'enterrement, en fin d'après-midi, alors que Roseline et ses neveux étaient encore chez Grann, je rejoignis Madan Olsen qui recevait dans sa cour et sur sa galerie des parents de Grann, des pasteurs, et des commères, tous gens des mornes ${ }^{43}$. Ma présence n'empêcha pas de faire état de désaccords. Le temps de mon terrain, on disait de moi que j'étais « quelqu'un des mornes (moun mòn) », « quelqu'un de chez Madan Olsen (moun Madan Olsen) ». D'ailleurs n'étais-je pas là présente au milieu d'eux? Madan Olsen parla sans restriction, depuis un an et demi j'étais à ses côtés et elle avait fait de moi une confidente et un pion dans l'établissement de son pouvoir de commerçante dans la région. On conversa à bâtons rompus du retrait du cercueil dont on rendit coupable Amélia. Le petit rassemblement, à mesure des histoires racontées, se transforma en son procès. Roseline ne fut même pas mentionnée. On semblait penser qu'elle n'avait été qu'une courroie de transmission et que l'unique responsable était Amélia. On s'attarda longuement sur son « mauvais caractère (movè karaktè) » et son arrogance, qui s'exprimaient, selon l'assemblée en présence, par son évitement des interactions avec les gens des mornes, son habituelle froideur et le manque de respect dont elle faisait preuve. Valeur cardinale en Haïti, le respect ${ }^{44}$ renvoie tout à la fois à l'affirmation d'égalité entre les membres d'une communauté et à l'hospitalité que l'on retrouve dans la formule dialoguée entre le visiteur et l'hôte visité « Honneur! - Respect! » et que l'on entend encore, en particulier en milieu rural ${ }^{45}$. La mère de Madan Olsen raconta avoir été traitée comme un «chien » par Amélia malgré ses relations amicales avec Pasteur Christ-Roi. Alors qu'elle était à Jérémie et qu'elle n'avait pas d'autre choix que d'y passer la nuit, elle dit avoir demandé l'hospitalité à Amélia, qui ne se serait pas montrée et aurait demandé à son domestique de lui préparer un couchage sur la terrasse de la maison. La mère de Madan Olsen ajouta: «Elle ne nous montre pas de respect, elle regarde les apparences (Li pa fè nou respè, li gade nou sou aparans) », auquel lui répondit un jeune homme " oui c'est ça! elle se sent supérieure (wi se sa! L'ap pran pòz sou moun) ». Un cousin de Grann

43. À la fin de l'enterrement, j'ai d'abord fait quelques allers-retours entre la maison de Grann et celle de Madan Olsen. Puis, voyant ce qui se tramait chez cette dernière, j'ai fait le choix d'y rester.

44. Sur cette valeur de respect en Haïti, et en particulier chez les jeunes dans la capitale, voir Chelsey Kivland (2020, p. 88-115).

45. «Honneur (Honè)! » entonne la personne qui prétend entrer dans la cour d'une maison, elle attendra d'entendre en réponse « Respect (Respè)! » pour s'avancer. 
narra l'histoire d'un des prétendants d'Amélia qui s'était rétracté après avoir constaté de manière répétée son égoïsme, ce qui provoqua l'hilarité du public. Madan Olsen, de son côté, rappela que Grann avait été un temps fidèle de l'église des blan. Pour elle, il n'y avait pas de doute, la délocalisation du cercueil exprimait l'hostilité d'Amélia à l'encontre des missionnaires américains : « elle ne s'entend pas avec [le missionnaire] (li pa byen avek li) ». Par ailleurs, en disant, avec une mimique de dégoût, que le cercueil avait fini « sous un arbre (anba pyebwa) » alors qu' " ils avaient eu le temps de faire une belle installation, de mettre le cercueil correctement (yo te gen tan fè bel enstalasyon, mete serkeyi korekteman) ", elle condamnait un emplacement inconvenant pour un événement qui devait, au contraire, laisser place aux marques de considération et à un cérémonial plus « correct » et flatteur. D'autant que, comme je l'ai dit plus haut, le moment de l'exposition du corps dans le cercueil est un des faits saillants des funérailles où la famille donne à voir son statut social. Enfin, elle signala à l'assemblée combien elle avait, tout au long de la maladie de Grann, donné de soi et de son temps: « Je fais des efforts, c'est que Madame est lourde! [...] je la baigne, je m'occupe d'elle, je la porte avec toutes mes forces (M'ap redi, Madanm lan lou! [...] M'ap benyen'l, m'ap okipe'l, m'konn pote'l avek tout fòs mwen) ». Vint ensuite la dénonciation de l'absence d'Amélia: « Je ne l'ai pas vue, pas même une fois. Elle n'a jamais demandé comment Madame allait. Elle n'a jamais appelé. Et c'est elle qui décide... (Mwen pat wè li, mèm yon jou. Li pat janm mande koman madanm nan ye. Li pat janm rele. Pou se li mèm ki te deside!) ». Madan Olsen confortait ainsi sa place dans la famille et ses liens avec Grann tandis qu'elle refusait, rétroactivement, à Amélia toute légitimité pour déterminer les contours de la famille et à prendre des décisions pour la famille. À aucun moment, les personnes présentes ne reprirent à leur compte la généalogie exposée par Amélia au cours de la cérémonie, laquelle généalogie, comme nous l'avons vu, étendait et incluait son père et sa propre personne à la famille de Grann. Ce dilatement des frontières de la famille non seulement n'était pas adopté par ceux présents mais il était même contesté: «Elle a donné un mauvais témoignage! Je ne comprends pas pourquoi elle a fait cette déclaration, messieurs (Li bay movè temwanyaj! M'pa konn sak fè li bay deklarasyon sa-a mesyè) » dit un hôte avec beaucoup d'emphase.

Il apparaît donc que l'hostilité de Madan Olsen envers Amélia ne se situait pas sur le plan théologique mais qu'elle n'était pas, pour autant, dépourvue de prétexte religieux. Ceux présents reprochaient à Amélia d'être pentecôtiste. On peut penser, en revanche, qu'Olsen, sa femme et sa belle-mère percevaient les agissements d'Amélia comme un affront à l'institution religieuse EBM à laquelle ils avaient consacré les quinze dernières années de leur vie. De manière plus générale, tous ceux présents dans la cour de Madan Olsen lui reprochaient d'avoir manqué de respect à la défunte, aux gens des mornes, et aux missionnaires. À travers cette question du respect, on voit finalement surgir une ligne 
de division entre les « personnes de l'étranger et de la capitale (moun andeyò) », adeptes du statu quo et d'actions coordonnées avec Amélia, et ceux des mornes qui, entre eux, font le réquisitoire de ceux du dehors. Cette distinction doit être lue à l'aune de l'histoire post-indépendance du pays au cours de laquelle s'est établie une fracture, alimentée par un pouvoir prédateur, entre la province (qualifiée de « pays en-dehors [peyi andeyò] »), dont les habitants sont supposément arriérés, et la capitale, siège de la civilisation (Barthélémy 1990). Cette fracture s'est complexifiée, sans pour autant disparaître, avec l'apparition des dyaspora (ceux qui ont résidé ou résident à l'étranger) ${ }^{46}$. Les attaques des gens des mornes à l'encontre d'Amélia sont proférées avec un plaisir évident (profusion de rires, histoires qui s'enchaînent, public joueur) fondé dans l'affinité et la concordance des opinions et des expériences et dans le fait de persifler et de se gausser des « puissants », qui sont qualifiés à leur tour, dans une inversion carnavalesque, de gens des mornes, où se discerne l'orgueil blessé des « plus petits».

\section{Conclusion}

Comme le remarque Marleen De Witte (2001, p. 68-69), il peut être parfois difficile de percevoir les conflits au cours des funérailles tant les convenances et les apparences doivent être préservées. Dans le cas qui nous occupe, les frictions furent contenues au cours de la cérémonie (à l'exemple de Pasteur Alexis qui adjura Olsen de se taire pour ne pas créer de « discussions ») ce n'est qu'en fin de cérémonie que l'aigreur se répandit à la faveur d'un public réduit à quelques individus qui partageaient des caractéristiques communes (gens des mornes, expérience passée de l'arrogance et de la mesquinerie d'Amélia), qui dans ces circonstances masquaient des différences sociales internes aux mornes ${ }^{47}$. Les migrations internes et externes au pays (à Mòn Anvè, on reçoit le titre de dyaspora que l'on soit parti à la ville ou à l'étranger) ont affecté en profondeur le paysage économique et démographique des mornes, et plus largement des différents milieux ruraux haïtiens. L'infinie gamme de positions sociales chez les « ruraux » (Moral 1978 [1961], p. 209-210; Ans 1987, p. 260; Bernardin 1991, p. 270-271) a connu de nouveaux facteurs de différenciation avec la présence de parents migrants dans les familles, tandis que la fracture entre peyi andeyò et capitale a été redoublée par celle entre lot bò dlo (de l'autre côté de l'eau: en dehors d'Haïti) et nan peyi a (au pays). La plus ou

46. Sur les différents usages du terme « diaspora » en Haïti, voir Joseph 2015 et sur la manière dont la ligne de division (en-dehors/de la ville) est activée en contexte de migration, voir Joseph 2019.

47. L'un des pasteurs présents au cours du «procès » est un petit paysan pauvre. Alors qu'Auguste, un neveu de Grann, ou encore Madan Olsen sont propriétaires d'un véhicule, de terres, et obtiennent des crédits de la banque pour commercer. 
moins grande participation de ces parents lointains dans l'économie familiale, rituelle et communautaire, leur venue saisonnière ou leur absence interminable, la communication et la mémoire faiblement ou intensément entretenues, tous ces éléments marquent concrètement la vie des mornes, les activités sociales et religieuses, les activités productives et de développement, les projets de vie des plus jeunes, les campagnes politiques locales, etc. Ce mouvement de migration, qui s'est intensifiée au long de ces trois dernières décennies, s'est accompagné d'une autre dynamique, non moins importante et qui en est souvent indissociable: celle de l'ascension évangélique et pentecôtiste. Cette indissociabilité provient du fait que les courants protestants aussi bien baptistes que pentecôtistes, et plus récemment néo-pentecôtistes, sont des phénomènes éminemment globaux, qui recomposent les relations entre le Sud et le Nord, entre les Suds entre eux et établissent de nouvelles circulations des « produits » religieux et culturels (Coleman et Hackett 2015; Wilkinson 2012). L'accroissement du nombre de leurs fidèles et leur diffusion accélérée doivent beaucoup aux migrants qui, en intégrant les Églises communautaires de leur groupe diasporique voient leur processus d'intégration dans la société d'accueil facilité et, conjointement, trouvent la sécurité et la possibilité de maintenir ou de revendiquer leur identité d'origine ${ }^{48}$. L'appartenance religieuse commune dans une « famille transnationale » peut aussi participer au renforcement des liens, à travers des prières collectives virtuelles, à travers l'envoi de textes, d'images, d'audiovisuels sur les réseaux sociaux, à travers des retrouvailles au cours d'un événement religieux dans le pays d'origine ou d'accueil. Il n'est pas impossible non plus que la conversion au protestantisme, lors d'une migration, produise l'effet inverse et distende les liens avec des parents pratiquants du vodou, par exemple, restés au pays. Dans les funérailles de Grann, on a pu voir comment le protestantisme évangélique et la parenté transnationale transforment par leurs actions conjointes le rituel funéraire - en en éliminant certains éléments (les pratiques rituelles qui se poursuivent après l'enterrement) ou en s'appuyant d'autres (il suffit de penser à la toilette mortuaire). L'agencement des deux dynamiques s'est opéré sur le mode de la conciliation, ou plutôt, sur celui de l'accord non-prémédité. Pensons, par exemple, au fait que le parent migrant ne disposant que de peu de temps pour participer aux funérailles, son départ dans les jours qui suivent l'enterrement s'accorde, dans le cas que l'on s'est proposé d'analyser, avec le fait que le rituel protestant des funérailles s'interrompt avec l'enterrement. On aurait pu imaginer que les deux dynamiques transformationnelles entrent en conflit mais les affinités qui lient l'une à l'autre peuvent avoir pour effet de limiter les courts-circuits.

Enfin, il apparaît plus que jamais nécessaire de sortir les campagnes haïtiennes des seules catégories de lecture que sont la petite paysannerie et le vodou (le plus

48. Sur ces questions dans la diaspora haïtienne, voir Louis 2015; Rey et Stepick 2013. 
souvent indissociablement liés) et de mieux rendre compte des conditions socio-économiques différenciées de leurs habitants - des conditions qui ont gagné de l'épaisseur et de nouveaux échelons sous l'action des dynamiques migratoires et religieuses - et des changements religieux qui n'ont pas attendu la fin $\mathrm{du} \mathrm{Xx}^{\mathrm{e}}$ siècle pour se profiler. La conversion au protestantisme évangélique et pentecôtiste et la migration constituent aujourd'hui, de fait, des opérateurs prééminents de structuration des différenciations sociales, des statuts sociaux et des relations de pouvoir dans les mornes. *

* Manuscrit reçu en mai 2020, accepté pour publication en avril 2021.

Remerciements - Cet article a bénéficié des commentaires avisés d'Ary Gordien, Delphine Manetta, Élise Capredon, Handerson Joseph et Rodrigo Bulamah; qu'ils en soient remerciés. Il a gagné sa forme finale grâce aux précieuses remarques des évaluateurs et de Philippe Erikson. Au cours de sa rédaction, j'ai bénéficié d'une bourse du programme Capes-Print (Brésil).

\section{Références citées}

AfFERgAn Francis

1975 «Esthétique de la mort et vie quotidienne aux Antilles », Traverses, 1, p. 81-88. Ans André-Marcel d'

1987 Haïti : paysage et société, Karthala, Paris.

Audebert Cédric

2017 «The recent geodynamics of haitian migration in the Americas: refugees or economic migrants? », Revista Brasileira de Estudos de População, 34 (1), p. $55-71$.

BARTHÉLÉMY Gérard

1990 L'univers rural haïtien. Le pays en dehors, L'Harmattan, Paris.

BASTIEN Rémy

1985 [1951] Le paysan haïtien et sa famille, ACCT/Karthala, Paris.

BERNARDIN Ernst

1991 L'espace rural haïtien. Bilan de 40 ans d'exécution des projets nationaux et internationaux de développement 1950-1990, Éditions des Antilles, Port-au-Prince.

Bidegain Gabriel

2013 «Les Haïtiens qui sont partis. Combien sont-ils? Où sont-ils? » [en ligne], version actualisée, janvier 2013, 31 p., http://classiques.uqac.ca/contemporains/ Bidegain_Gabriel/Haitiens_qui_sont_partis/Haitiens_qui_sont_partis_texte. html, consulté le 30/07/2021.

BLoch Maurice et Jonathan PARRY

1982 «Introduction: death and the regeneration of life », in Maurice Bloch et Jonathan Parry (dir.), Death and the Regeneration of Life, Cambridge University of Press, Cambridge. 
BOURDiEu Pierre

2002 Le bal des célibataires. Crise de la société paysanne en Béarn, Éditions du Seuil (Points), Paris.

BrazIEL Jane

2017 Riding with Death. Vodou Art and Urban Ecology in the Streets of Port-auPrince, University Press of Mississippi, Jackson.

Brodwin Paul

2003 «Pentecostalism in translation. Religion and the production of community in the Haitian diaspora », American Ethnologist, 1 (30), p. 85-101.

Bulamah Rodrigo C.

2013 «O cultivo dos comuns: parentesco e práticas sociais em Milot, Haiti », dissertation de master en anthropologie, Unicamp, Campinas.

Candea Matei, Joanna Cook, Catherine Trundle et Thomas Yarrow (dir.)

2015 Detachment. Essays on the limits of relational thinking, Manchester University Press, Manchester.

CAsey Matthew

2017 Empire's Guestworkers. Haitian Migrants in Cuba during the Age of US Occupation, Cambridge University Press, New York.

Charlier Philippe

2015 Zombis, enquête chez les morts vivants, Éditions Tallandier, Paris.

Clorméus Lewis Ampidu

2014 «L'Église catholique face à la diversité religieuse à Port-au-Prince (19422012) », Archives de sciences sociales des religions, 166, p. 157-180.

Coleman Simon et Rosalind Hackett

2015 The Anthropology of Global Pentecostalism and Evangelicalism, New York University Press, New York.

Comhaire-Sylvain Suzanne

1959 « Mort et funérailles dans la région de Kenscoff (Haïti) », Revue de l'Institut de Sociologie, 2, p. 197-232.

Conway Frederick

1978 « Pentecostalism in the context of Haitian religion and health practice », thèse de doctorat en anthropologie, American University, Washington (DC).

CORTEN André

2001 Misère, religion et politique en Haïti, Karthala, Paris.

Cosentino Donald (dir.)

2012 In Extremis. Death and Life in 21st-century Haitian Art, Fowler Museum at UCLA, Los Angeles.

2018 «From zombi to Samdi: late transformations in Haitian eschatology », in Maarit Forde et Yanique Hume (dir.), Passages \& Afterworlds. Anthropological Perspectives on Death in the Caribbean, Duke University Press, Durham/ London, p. 159-175.

Crousaz Karine

2013 «La mort, les funérailles et l'au-delà: la rupture de la Réforme en Suisse romande », in Dave Lüthi (dir.), Le Marbre et la poussière. Le patrimoine 
Des funérailles évangéliques dans une famille transnationale haïtienne

funéraire de la Suisse romande (XIV -XVIII $I^{e}$ siècles), Cahiers d'archéologie romande, Lausanne, vol. 1, p. 65-76.

Dalmaso Flavia

2018 «Heranças de família: terras, pessoas e espiritos no sul do Haiti », Mana, 24 (3), p. 96-123.

DAmus Obrillant

2020 «Le rôle des matrones dans la gestion et l'utilisation durable de la biodiversité en Haïti. Matrimonialisation et sauvegarde de leur métier », Études caribéennes, 45, p. 1-20.

De WitTe Marleen

2001 Long Live the Dead! Changing Funeral Celebrations in Asante, Ghana, Aksant Academic Publishers, Amsterdam.

2003 « Money and death. Funeral business in Asante, Ghana », Africa. Journal of the International African Institute, 73 (4), p. 531-559.

Debien Gabriel

1974 «Petits cimetières de quartier et de plantation à Saint-Domingue au $\mathrm{XVIII}^{\mathrm{e}}$ siècle », Revue française d'histoire d'outre-mer, 225, p. 522-541.

Desmangles Leslie G.

1992 The Faces of the Gods. Vodou and Roman Catholicism in Haiti, University of North Carolina Press, Chapel Hill/London.

DIRKSEN Rebecca

2020 After the Dance, the Drums are Heavy. Carnival, Politics, and Musical Engagement in Haiti, Oxford University Press, New York.

FARMER Paul

1992 Aids and Accusation. Haiti and the Geography of Blame, University of California Press, Berkeley.

FER Yannick

2005 «Genèse des émotions au sein des Assemblées de Dieu polynésiennes », Archives de sciences sociales des religions, 131-132, p. 143-163.

Fog Olwig Karen

2002 " A wedding in the family: home making in a global kin network », Global Networks, 2 (3), p. 1470-2266.

2009 «A proper funeral: contextualizing community among Caribbean migrants », Journal of the Royal Anthropological Institute, 15, p. 520-537.

Fouron Georges

2020 «Haiti's painful evolution form promised land to migrand-sending nation », The online Journal of the Migration Policy Institute [en ligne], 19 août, https://www.migrationpolicy.org/article/haiti-painful-evolution-promisedland-migrant-sending-nation, consulté le 30/07/2021.

Grosse Christian

2019 «Une mort déritualisée? Usages funéraires dans la tradition protestante réformée ", in Jean-François Boudet (dir.), Les rites et usages funéraires. Essais d'anthropologie juridique, Presses universitaires d'Aix-Marseille, Aix-en-Provence, p. 181-196. 
Herskovits Melville J.

2012 [1937] Life in a Haitian Valley, Markus Wiener Publishers, Princeton.

Horst Heather

2004 «A pilgrimage home. Tombs, burial and belonging in Jamaica », Journal of Material Culture, 9 (1), p. 11-26.

Horst Heather et David Miller

2005 « From cell phone to link-up: cell phones and social networking in Jamaica », Current Anthropology, 46 (5), p. 755-778.

HURBON Laënnec

1993 Les mystères du vodou, Gallimard, Paris.

2001 «Pentecostalism and transnationalisation in the Caribbean », in André Corten et Ruth Marshall-Fratani (dir.), Between Babel and Pentecost. Transnational Pentecostalism in Africa and Latin America, Hurst and Company, London, p. 124-141.

JINDRA Michael et Joël NORET

2011 «African funerals and sociocultural change: a review of momentous transformations across a continent », in Michael Jindra et Joël Noret (dir.), Funerals in Africa. Explorations of a Social Phenomenon, Berghahn Books, New York/ Oxford, p. 16-40.

JOSEPH Handerson

2015 «Diaspora. As dinâmicas da mobilidade haitiana no Brasil, no Suriname e na Guiana francesa », thèse de doctorat en anthropologie, Museu Nacional (UFRJ), Rio de Janeiro.

2019 «Mobilité transfrontalière haïtienne au Brésil: kongo, vyewo et dyaspora », in Béchacq Dimitri, Calixte Fritz et Meudec Marie, Les migrations et la Caraïbe. (Dés) ancrages, mouvements et contraintes, L'Harmattan (Recherches Haïtiano-Antillaises, 8), Paris, p. 207-231.

2020 «Maisons diasporas et maisons locales. Mobilités haïtiennes et réseaux transnationaux », Etnográfica, 24 (3), p. 749-774.

KERBOULl Jean

1973 Le Vaudou. Magie ou religion?, Robert Laffont, Paris.

Kivland Chelsey

2020 Street Sovereigns. Young Men and the Makeshift State in Urban Haiti, Cornell University Press, Ithaca/London.

LAGUERRE Michel

1978 «Ticouloute and his kinsfolk: the study of a Haitian extended family », in Demitri B. Shimkin, Edith M. Shimkin et Dennis A. Frate (dir.), The Extended Family in Black Societies, Mouton, The Hague, p. 406-445.

1998 Diasporic Citizenship. Haitian Americans in Transnational America, St. Martin's Press, New York.

LANGEWIESCHE Katrin

2003 Mobilité religieuse. Changements religieux au Burkina Faso, Lit Verlag, Münster. LAROSE Serge

1994 « Religion et politique en Haïti », in Françoise-Romaine Ouellette et Claude Bariteau (dir.), Entre tradition et universalisme, Institut québécois de recherche sur la culture, Québec, p. 117-134. 
Des funérailles évangéliques dans une famille transnationale haïtienne

LAURENT Pierre-Joseph

2018 Amours pragmatiques. Familles, migrations et sexualité au Cap-Vert aujourd'hui, Karthala, Paris.

LouIs JR. Bertin M.

2015 My Soul is in Haiti. Protestantism in the Haitian Diaspora of the Bahamas, New York University Press, New York/London.

LOWENTHAL Ira P.

1987 «'Marriage is 20, children are 21': the cultural construction of conjugality and the family in rural Haiti », thèse de doctorat en anthropologie, Johns Hopkins University, Baltimore.

Mayrargue Cédric

2009 «Pluralisation et compétition religieuses en Afrique subsaharienne. Pour une étude comparée des logiques sociales et politiques du christianisme et de l'Islam », Revue Internationale de Politique Comparée, 16 (1), p. 83-98.

Mazzucato Valentina, Mirjam KabKi et Lothar Smith

2006 «Transnational migration and the economy of funerals: changing practices in Ghana », Development and Change, 37 (5), p. 1047-1072.

McAlister Elizabeth

2002 Rara! Vodou, Power, and Performance in Haiti and its Diaspora, University of California Press, Berkeley.

2013 «Humanitarian adhocracy, transnational new apostolic missions, and evangelical anti-dependency in a Haitian refugee camp », Nova Religio. The Journal of Alternative and Emergent Religions, 16 (4), p. 11-34.

McCARThy Brown Karen

2001 [1991] Mama Lola. A Vodou Priestess in Brooklyn, University of California Press, Berkeley.

MétrauX Alfred

1953 «Vodou et protestantisme », Revue de l'histoire des religions, 144 (2), p. 198-216.

1954 « Rites funéraires des paysans haïtiens », Arts et traditions populaires, 2 (4), p. 289-306.

1958 Le vaudou haïtien, Gallimard, Paris.

MÉzié Nadège

2016 «Emergência e ascensão dos protestantismos no Haiti: um panorama histórico », Debates do NER, 17 (29), p. 289-327.

2019a «O ultimo encontro: o pastor e sua amiga "cabeça dura" ", Debates do NER, 19 (35), p. 405-414.

2019 b « Kretyen sou aparans: diferenciação e processos morais entre evangélicos

Moral Paul no Haiti », Anthroplógicas, 23 (30), p. 38-68.

1978 [1961] Le paysan haïtien, Éditions Fardin, Port-au-Prince.

Mullins Mark R.

1990 « Japanese pentecostalism and the world of the dead: a study of cultural adaptation in Iesu no Mitama Kyokai », Japanese Journal of Religious Studies, 17 (4), p. 353-374. 
Murray Gérald F.

1977 « The evolution of Haitian peasant land tenure: a case study in agrarian adaptation to population growth », thèse de doctorat en anthropologie, Columbia University, New York.

Noret Joël

2004 «De la conversion au basculement de la place des morts. Les défunts, la personne et la famille dans les milieux pentecôtistes du Sud-Bénin », Politique africaine, 1 (93), p. 143-155.

2014 «Sur le dos des morts? Organiser des funérailles catholiques à Abomey (Bénin)», Terrain, 62, p. 54-69.

2017 «Sociologie actuelle des funérailles. Prise en charge de la mort et espace social dans l'Afrique d'aujourd'hui », Cahiers d'Études africaines, 4 (228), p. 1011-1033.

Opas Minna

2019 «Keeping Boudaries in motion: Christian denominationalism and sociality in Amazonia », Anthropological Quarterly, 92 (4), p. 1069-1097.

Plaza Dwaine et Lauren Plaza

2019 «Facebook and WhatsApp as elements in transnational care chains for the Trinidadian diaspora », Genealogy, 3 (15), p. 1-20.

REY Terry et Alex STEPICK

2013 Crossing the Water and Keeping the Faith. Haitian Religion in Miami, New York University Press, New York.

RichMAN Karen

2012 « Religion at the epicenter: agency and affiliation in Léogâne after the earthquake ", Studies in Religion/Sciences Religieuses, 41 (2), p. 148-165.

2018 [2005] Migration and Vodou, University Press of Florida, Gainesville.

2018 "Mortuary rites and social dramas in Léogâne, Haiti », in Maarit Forde et Yanique Hume (dir.), Passages \& Afterworlds. Anthropological Perspectives on Death in the Caribbean, Duke University Press, Durham/London.

Romain Charles-Poisset

2004 [1986] Le protestantisme dans la société haïtienne. Contribution à l'étude historique, sociographique et descriptive d'une religion, Presses de l'université Jean Price-Mars/Educa Vision Inc., Coconut Creek.

Rus Alin

2014 « Two different burial rituals in the same village of Romania: Helesteni Iasi », in Marius Rotar, Adriana Teodorescu et Corina Rotar (dir.), Dying and Death in 18th-21st Century Europe. Volume 2, Newcastle upon Tyne, Cambridge Scholars Publishing, p. 60-85.

SCHILLER Nina Glick

1999 «Transmigrants and nation-states: something old and something new in the U.S. immigrant experience », in Charles Hirschman, Philip Kasinitz et Josh DeWind (dir.), The Handbook of International Migration. The American Experience, Russell Sage, New York, p. 94-119. 
Des funérailles évangéliques dans une famille transnationale haïtienne

Sмiтh Daniel Jordan

2004 «Burials and belonging in Nigeria: rural-urban relations and social inequality in a contemporary African ritual », American Anthropologist, 106 (3), p. 569-579.

SмIтн Katherine

2010 «Dialoging with the urban dead in Haiti: manifestations of Gede in an age of crisis », Southern Quaterly, 47 (4), p. 61-90.

2012 «Genealogies of Gede », in Donald Cosentino (dir.), In Extremis. Death and Life in 21st-century Haitian Art, Fowler Museum at UCLA, Los Angeles, p. 85-99.

TREMBLAY Johanne

1995 Mères, pouvoir et santé en Haïti, Karthala, Paris.

VAN DER PiJl Yvon

2016 « Death in the family revisited: ritual expression and controversy in a creole transnational mortuary sphere », Ethnography, 17 (2), p. 147-167.

VIDAL Claudine

1991 Sociologie des passions (Côte d'Ivoire, Rwanda), Karthala, Paris.

VONARX Nicolas

2008 «Vodou et pluralisme médico-religieux en Haïti », Anthropologie et Sociétés, 32 (3), p. 213-231.

WiLKinson Michael (dir.)

2012 Global Pentecostal Movements. Migration, Mission, and Public Religion, Brill, Leiden/Boston.

Woods Orlando

2012 «Sri Lanka's informal religious economy: evangelical competitiveness and Buddhist hegemony in perspective », Journal for the Scientific Study of Religion, 51 (2), p. 203-219.

Woodson Drexel G.

1990 Tout Mounn se Mounn, Men tout Mounn pa Menm: Microlevel Sociocultural Aspects of Land Tenure in a Northern Haitian Locality, thèse de doctorat en anthropologie, Chicago University, Chicago. 
